\title{
Modeling the Landau-Gauge Ghost Propagator in 2, 3 and 4 Space-Time Dimensions
}

\author{
Attilio Cucchieri, ${ }^{1, *}$ David Dudal, ${ }^{2,3, \dagger}$ Tereza Mendes, ${ }^{1, \ddagger}$ and Nele Vandersickel ${ }^{3, \S}$ \\ ${ }^{1}$ Instituto de Física de São Carlos, Universidade de São Paulo, Caixa Postal 369, 13560-970, São Carlos, SP, Brazil \\ ${ }^{2}$ KU Leuven Campus Kortrijk - KULAK, Department of Physics, Etienne Sabbelaan 53, 8500 Kortrijk, Belgium \\ ${ }^{3}$ Ghent University, Department of Physics and Astronomy, Krijgslaan 281-S9, 9000 Gent, Belgium
}

(Dated: February 5, 2016)

\begin{abstract}
We present an analytic description of numerical results for the ghost propagator $G\left(p^{2}\right)$ in minimal Landau gauge on the lattice. The data were produced in the $\mathrm{SU}(2)$ case using the largest lattice volumes to date, for $d=2,3$ and 4 space-time dimensions. Our proposed form for $G\left(p^{2}\right)$ is derived from the one-loop relation between ghost and gluon propagators, considering a tree-level ghost-gluon vertex and our previously obtained gluon-propagator results [1]. Although this one-loop expression is not a good description of the data, it leads to a one-parameter fit of our ghost-propagator data with a generally good value of $\chi^{2} / d o f$, comparable to other fitting forms used in the literature. At the same time, we present a simple parametrization of the difference between the lattice data and the one-loop predictions.
\end{abstract}

\footnotetext{
*attilio@ifsc.usp.br

$\dagger$ david.dudal@kuleuven.be

* mendes@ifsc.usp.br

$\S$ nele.vandersickel@ugent.be
} 


\section{INTRODUCTION}

An analytic description of propagators and vertices of Yang-Mills theories — at the nonperturbative level, in a given gaugeis a possible starting point for understanding the relevant features of these theories and, in particular, the phenomenon of color confinement [2-5]. From this point of view, the first natural step is the study of the infrared (IR) behavior of the gluon propagator $D\left(p^{2}\right)$ and of the ghost propagator $G\left(p^{2}\right)$ as functions of the momentum $p$. In the last thirty years, many numerical and analytic studies have addressed this issue in Landau gauge, in two, three and four space-time dimensions (see, for example, the reviews [5-10] and references therein). All the numerical studies, usually done for pure SU(2) and SU(3) lattice gauge theories, now agree that, in three and in four space-time dimensions [1, 11-21], the gluon propagator is IR-finite and the ghost propagator is free-like in the same limit. On the contrary, in the $2 d$ case $[1,14,15,18,22,23]$, the gluon propagator goes to zero at small momenta and the ghost propagator is IR-enhanced. In the former case, the numerical data can be related to the so-called massive solution of the Dyson-Schwinger equations [24-31], while in the latter case one should refer instead to the so-called scaling solution of these equations [2, 32-38]. The two different types of solutions can also be related to the Gribov-Zwanziger (GZ) [39-45] and Refined GZ (RGZ) [46-52] approaches, which correspond respectively to the scaling and to the massive behaviors for the gluon and ghost propagators in the deep IR limit. ${ }^{1}$

In Refs. [1, 18] we have presented an analytic description of lattice data [12,14] for the SU(2) Landau-gauge gluon propagator $D\left(p^{2}\right)$ in two, three and four space-time dimensions $d$. For the cases $d=3$ and 4 , the numerical data can be well fitted using tree-level predictions of the RGZ approach, i.e. considering sums of propagators of the type $\alpha /\left(p^{2}+\omega^{2}\right)$, where $\alpha$ and $\omega$ are in general complex constants. ${ }^{2}$ On the contrary, in the $2 d$ case, no such predictions are available [48], and the data may be fitted using a noninteger power of $p$ in the numerator of $D\left(p^{2}\right)$. These fitting forms have subsequently been used in Ref. [64] to evaluate the one-loop-corrected ghost propagator $G\left(p^{2}\right)$ and to analyze the behavior of the so-called Gribov ghost form factor $\sigma\left(p^{2}\right)$, defined by

$$
G\left(p^{2}\right)=\frac{1}{p^{2}} \frac{1}{1-\sigma\left(p^{2}\right)}
$$

i.e.,

$$
\sigma\left(p^{2}\right) \equiv 1-\left[p^{2} G\left(p^{2}\right)\right]^{-1}
$$

Using these analytic results one can show that, considering the bare coupling constant $g^{2}$ as a free parameter, the massive solution $G\left(p^{2}\right) \sim 1 / p^{2}$, corresponding to $\sigma(0)<1$, is obtained for all values of $g^{2}$ smaller than a "critical" value $g_{c}^{2}$. At $g_{c}^{2}$, one has $\sigma(0)=1$ and the ghost propagator is IR-enhanced. These findings confirm that, in the Dyson-Schwinger-equation approach, the ghost propagator admits a one-parameter family of behaviors [65-68], labeled by the coupling constant $g^{2}$.

In this work we present the final step of our analysis, using the one-loop results for $G\left(p^{2}\right)$ of Ref. [64] as theoretical predictions for the analytic modeling of numerical data $[12,15]$ for the ghost propagator in Landau gauge in two, three and four space-time dimensions. (Similar studies have been presented in Refs. [69-71] for the four-dimensional case.) We find that the proposed analytic forms do not yield a good description of the ghost-propagator data. This is in agreement with Refs. [69-71]. Nevertheless, by treating $g^{2}$ as a free parameter in these forms, one obtains fits of $G\left(p^{2}\right)$ with generally good values of $\chi^{2} / d o f$, comparable to other fitting forms used in the literature (see e.g. $[15,27]$ ). Finally, we attempt a simple parametrization of the difference between the lattice data and the one-loop predictions, which turns out to be very similar for the $d=2,3$ and 4 cases. This supports a possible interpretation of the physical effects that are missing in the one-loop results [69-72].

The paper is organized as follows. In the next section, we recall the main results of Refs. [1, 18, 64] and, in particular, the formulae used in our analysis of the ghost propagator. Then, in Section III, we present and discuss the fits to the lattice data. Lastly, in Section IV, we outline our conclusions.

\footnotetext{
${ }^{1}$ The interested reader should see Refs. [53-63] for other approaches and points of view on the scaling and/or the massive solutions.

${ }^{2}$ Let us mention that this proposed behavior for the gluon propagator, i.e. a pole structure with complex-conjugate masses (with comparable real and imaginary parts), can be interpreted as describing an unstable particle. This is discussed in Ref. [1], where we also compute the resulting mass and decay width for the gluon in the $4 d$ case.
} 


\section{ONE-LOOP PREDICTIONS}

As already explained in the Introduction, in Refs. $[1,18]$ the $\mathrm{SU}(2)$ gluon propagator has been fitted in $d=3,4$ and 2 using, respectively, the functions ${ }^{3}$

$$
\begin{gathered}
D\left(p^{2}\right)=C \frac{\left(p^{2}+s\right)\left(p^{2}+1\right)}{\left(p^{4}+u^{2} p^{2}+t^{2}\right)\left(p^{2}+k\right)}, \\
D\left(p^{2}\right)=C \frac{p^{2}+s}{p^{4}+u^{2} p^{2}+t^{2}}
\end{gathered}
$$

and

$$
D\left(p^{2}\right)=C \frac{p^{2}+l p^{\eta}+s}{p^{4}+u^{2} p^{2}+t^{2}}
$$

The first two propagators are tree-level expressions obtained in the RGZ approach [46-52], while the last formula is a simple generalization of the form in Eq. (4). Note that these three functions can be expressed as linear combinations of propagators of the type $1 /\left(p^{2}+\omega^{2}\right)$, where $\omega^{2}$ is in general a complex number. In particular, Eqs. (3) and (4) can be re-written respectively as

$$
D\left(p^{2}\right)=\frac{\alpha}{p^{2}+\omega_{1}^{2}}+\frac{\beta}{p^{2}+\omega_{2}^{2}}+\frac{\gamma}{p^{2}+\omega_{3}^{2}}
$$

and

$$
D\left(p^{2}\right)=\frac{\alpha_{+}}{p^{2}+\omega_{+}^{2}}+\frac{\alpha_{-}}{p^{2}+\omega_{-}^{2}} .
$$

The fits to the data $[1,18]$ suggest that, in the $3 d$ case [see Eq. (6)], one root is real, for example $\omega_{1}$, while the other two roots are complex-conjugate, i.e. $\left(\omega_{2}^{2}\right)^{*}=\omega_{3}^{2}$, implying also $\beta=\gamma^{*}$. Similarly, in the $4 d$ case [see Eq. (7)] one finds, by fitting the lattice data, that $\omega_{ \pm}^{2}$ are complex-conjugate roots, i.e. $\omega_{-}^{2}=\left(\omega_{+}^{2}\right)^{*}$ and $\alpha_{-}=\alpha_{+}^{*}$. On the other hand, in the $2 d$ case we need to consider the more general form $p^{\eta} /\left(p^{2}+\omega^{2}\right)$ with $\eta \geq 0$. Indeed, one can re-write Eq. (5) as

$$
D\left(p^{2}\right)=\frac{\alpha_{+}+i c p^{\eta}}{p^{2}+\omega_{+}^{2}}+\frac{\alpha_{-}-i c p^{\eta}}{p^{2}+\omega_{-}^{2}}
$$

where $c$ is real, $\alpha_{-}=\alpha_{+}^{*}$ and $\omega_{-}^{2}=\left(\omega_{+}^{2}\right)^{*}$. Estimates for the fitting parameters of the functions (3)-(8) can be found, respectively, in Tables IX, II, XIII, XI, IV and XIV of Ref. [1].

Using the notation of Ref. [64], the one-loop-corrected Landau-gauge ghost propagator can be evaluated [for the $\mathrm{SU}\left(N_{c}\right)$ gauge group in the $d$-dimensional case] using the relation

$$
G\left(p^{2}\right)=\frac{1}{p^{2}}-\frac{\delta^{a b}}{N_{c}^{2}-1} \frac{1}{p^{4}} g^{2} f^{a d c} f^{c d b} \int \frac{\mathrm{d}^{d} q}{(2 \pi)^{d}}(p-q)_{\mu} p_{v} D\left(q^{2}\right) P_{\mu v}(q) \frac{1}{(p-q)^{2}},
$$

where $\delta^{a b} D\left(q^{2}\right) P_{\mu v}(q)$ stands for the gluon-propagator forms described above and $P_{\mu v}(q)=\left(\delta_{\mu \nu}-q_{\mu} q_{v} / q^{2}\right)$ is the usual projector onto the transverse sub-space. Here we have considered the tree-level ghost-gluon vertex $i g f^{a d c} p_{v}$, where $p$ is the outgoing ghost momentum. The color indices $a, d, c$ refer, respectively, to the incoming ghost, to the gluon and to the outcoming ghost. Then, using for the gluon propagator the expressions (6)-(8) above and writing $G\left(p^{2}\right)$ as in Eq. (1), one can show [64] that the Gribov ghost form factor $\sigma\left(p^{2}\right)$ is given in three, four and two space-time dimensions by the formulae reported in the subsections below.

\footnotetext{
${ }^{3}$ Note that, here and in the following, we choose to present results for the $3 d$ and $4 d$ cases before the $2 d$ case.
} 


\section{A. The three-dimensional case}

Assuming (see above) that $\omega_{1}$ and $\alpha$ are real and writing the remaining fitting parameters of Eq. (6) as

$$
\beta=a+i b, \quad \gamma=a-i b
$$

and

$$
\omega_{2}^{2}=v+i w, \quad \omega_{3}^{2}=v-i w
$$

we obtain [64]

$$
\sigma_{1 L}\left(p^{2}\right)=\frac{g^{2} N_{c}}{8}\left[\frac{\alpha s\left(p^{2}, \omega_{1}^{2}\right)}{4 \pi \omega_{1}^{2} p^{3}}+f_{R}\left(p^{2}\right)\right]
$$

where

$$
\begin{gathered}
s\left(p^{2}, \omega^{2}\right)=-\pi p^{4}+2 p^{3} \sqrt{\omega^{2}}-2 p\left(\omega^{2}\right)^{3 / 2}+2\left(p^{2}+\omega^{2}\right)^{2} \arctan \left(\frac{p}{\sqrt{\omega^{2}}}\right), \\
f_{R}\left(p^{2}\right)=f_{1}\left(p^{2}\right)+f_{2}\left(p^{2}\right)+f_{3}\left(p^{2}\right)+f_{4}\left(p^{2}\right)+f_{5}\left(p^{2}\right)
\end{gathered}
$$

with

$$
\begin{aligned}
R & =\sqrt{v^{2}+w^{2}} \\
f_{1}\left(p^{2}\right) & =-p \frac{a v+b w}{2 R^{2}} \\
f_{2}\left(p^{2}\right) & =\frac{(a v+b w) \sqrt{R+v}-(b v-a w) \sqrt{R-v}}{\sqrt{2} \pi R^{2}} \\
f_{3}\left(p^{2}\right) & =-\frac{1}{p^{2}} \frac{a \sqrt{R+v}-b \sqrt{R-v}}{\sqrt{2} \pi} \\
f_{4}\left(p^{2}\right) & =A\left(p^{2}\right) \frac{p^{4}(a v+b w)+2 a p^{2} R^{2}+R^{2}(a v-b w)}{2 \pi R^{2} p^{3}} \\
f_{5}\left(p^{2}\right) & =-L\left(p^{2}\right) \frac{p^{4}(b v-a w)+2 b p^{2} R^{2}+R^{2}(b v+a w)}{2 \pi R^{2} p^{3}}
\end{aligned}
$$

and

$$
\begin{aligned}
& A\left(p^{2}\right)= \begin{cases}\arctan \left(\frac{\sqrt{2} p \sqrt{R+v}}{R-p^{2}}\right) & \text { if } R-p^{2}>0 \\
\pi+\arctan \left(\frac{\sqrt{2} p \sqrt{R+v}}{R-p^{2}}\right) & \text { if } R-p^{2}<0\end{cases} \\
& L\left(p^{2}\right)=\ln \left[\frac{\sqrt{p^{4}+2 p^{2} v+R^{2}}}{R+p(p+\sqrt{2} \sqrt{R-v})}\right]
\end{aligned}
$$

\section{B. The four-dimensional case}

By working in the $\overline{\mathrm{MS}}$ scheme, using dimensional regularization and writing the fitting parameters of Eq. (7) as $\alpha_{ \pm}=a \pm i b$ and $\omega_{ \pm}^{2}=v \pm i w$, one finds [64]

$$
\sigma_{1 L}^{\overline{\mathrm{MS}}}\left(p^{2}\right)=\frac{g^{2} N_{c}}{32 \pi^{2} R^{2}}\left[-p^{2} t_{1}\left(p^{2}\right)+R^{2} t_{2}\left(p^{2}\right)+p^{-2} t_{3}\left(p^{2}\right)-p^{-4} t_{4}\left(p^{2}\right)\right]
$$


with $R$ defined in Eq. (15),

$$
\begin{aligned}
& t_{1}\left(p^{2}\right)=(a v+b w)\left[\ell_{2}\left(p^{2}\right)+\ell_{3}\left(p^{2}\right)\right]-(b v-a w)\left[a_{1}\left(p^{2}\right)-a_{2}\left(p^{2}\right)\right], \\
& t_{2}\left(p^{2}\right)=a\left[5+\ell_{1}\left(p^{2}\right)+\ell_{2}\left(p^{2}\right)+\ell_{3}\left(p^{2}\right)-4 \ell_{4}\left(p^{2}\right)\right]-b\left[a_{1}\left(p^{2}\right)-a_{2}\left(p^{2}\right)-4 a_{3}\left(p^{2}\right)\right], \\
& t_{3}\left(p^{2}\right)=\left[1-3 \ell_{3}\left(p^{2}\right)\right]\left(a v^{3}-b w v^{2}+v a w^{2}-b w^{3}\right)-3 a_{2}\left(p^{2}\right)\left(b v^{3}+a w v^{2}+v b w^{2}+a w^{3}\right), \\
& t_{4}\left(p^{2}\right)=\ell_{3}\left(p^{2}\right)\left(a v^{4}-2 w b v^{3}-2 v b w^{3}-a w^{4}\right)+a_{2}\left(p^{2}\right)\left(b v^{4}+2 a w v^{3}+2 v a w^{3}-b w^{4}\right)
\end{aligned}
$$

and

$$
\begin{aligned}
& \ell_{1}\left(p^{2}\right)=\ln \left(\frac{p^{2}}{\bar{\mu}^{2}}\right), \\
& \ell_{2}\left(p^{2}\right)=\ln \left(\frac{R}{p^{2}}\right), \\
& \ell_{3}\left(p^{2}\right)=\ln \left(\frac{\sqrt{R^{2} p^{4}+R^{4}+2 v R^{2} p^{2}}}{R^{2}}\right), \\
& \ell_{4}\left(p^{2}\right)=\ln \left(\frac{\sqrt{p^{4}+2 v p^{2}+R^{2}}}{\bar{\mu}^{2}}\right), \\
& a_{1}\left(p^{2}\right)=\arctan \left(\frac{w}{v}\right), \\
& a_{2}\left(p^{2}\right)=\arctan \left(\frac{w p^{2}}{R^{2}+v p^{2}}\right), \\
& a_{3}\left(p^{2}\right)=\arctan \left(\frac{w}{v+p^{2}}\right) .
\end{aligned}
$$

The above result for $\sigma_{1 L}^{\overline{\mathrm{MS}}}\left(p^{2}\right)$ cannot, however, be directly compared to the lattice data, since the $\overline{\mathrm{MS}}$ scheme is defined only at the perturbative level. Thus, in order to make this comparison in the next section, we use a momentum-subtraction (MOM) renormalization scheme defined by

$$
\left.D^{\mathrm{MOM}}\left(p^{2}\right)\right|_{p^{2}=\bar{\mu}^{2}}=\frac{1}{\bar{\mu}^{2}},\left.\quad G^{\mathrm{MOM}}\left(p^{2}\right)\right|_{p^{2}=\bar{\mu}^{2}}=\frac{1}{\bar{\mu}^{2}} .
$$

The MOM-scheme condition for the gluon propagator affects only the global multiplicative factor $C$ in Eq. (4), or the parameters $\alpha_{ \pm}$in Eq. (7). As a consequence [see Eqs. (23)-(34)] the quantity $\sigma_{1 L}^{\overline{\mathrm{MS}}}\left(p^{2}\right)$ also gets modified by a global factor. At the same time, we can transform the above $\overline{\mathrm{MS}}$ result for $G\left(p^{2}\right)$ into the MOM scheme by writing ${ }^{4}$

$$
G^{\mathrm{MoM}}\left(p^{2}\right)=\frac{1}{p^{2}}\left[1-\sigma_{1 L}^{\overline{\mathrm{MS}}}\left(p^{2}\right)+h\left(\bar{\mu}^{2}\right)\right]^{-1},
$$

where the parameter $h\left(\bar{\mu}^{2}\right)$ is fixed by imposing the MOM-scheme condition (35), i.e.

$$
\sigma_{1 L}^{\overline{\mathrm{MS}}}\left(\bar{\mu}^{2}\right)=h\left(\bar{\mu}^{2}\right) .
$$

\section{The two-dimensional case}

In the $2 d$ case one finds [64]

$$
\sigma_{2 d}\left(p^{2}\right)=g^{2} N_{c}\left[\alpha_{+} f\left(p^{2}, \omega_{+}^{2}\right)+\alpha_{-} f\left(p^{2}, \omega_{-}^{2}\right)+i c \widetilde{f}\left(p^{2}, \omega_{+}^{2}, \eta\right)-i c \widetilde{f}\left(p^{2}, \omega_{-}^{2}, \eta\right)\right]
$$

\footnotetext{
${ }^{4}$ This corresponds to a one-loop (finite) shift in the renormalization factor of the ghost propagator.
} 
with

$$
f\left(p, \omega^{2}\right)=\frac{1}{8 \pi}\left[\frac{1}{p^{2}} \ln \left(1+\frac{p^{2}}{\omega^{2}}\right)+\frac{1}{\omega^{2}} \ln \left(1+\frac{\omega^{2}}{p^{2}}\right)\right]
$$

and

$$
\widetilde{f}\left(p, \omega^{2}, \eta\right)=\frac{\left(\omega^{2}\right)^{\eta / 2}}{4 \pi \eta p^{2}}\left[\frac{p^{2}+\omega^{2}}{\omega^{2}} B\left(\frac{\omega^{2}}{p^{2}+\omega^{2}} ; 1-\frac{\eta}{2}, 1+\frac{\eta}{2}\right)-B\left(1-\frac{\eta}{2}, 1+\frac{\eta}{2}\right)\right] .
$$

Here,

$$
B(x ; a, b)=\int_{0}^{x} \mathrm{~d} t t^{a-1}(1-t)^{b-1}
$$

is the incomplete Beta function, which is defined for $a, b>0$ [73], implying $2>\eta$ in our case, and $B(a, b) \equiv B(1 ; a, b)$ is the Beta function. Then, by writing $\alpha_{ \pm}=a \pm i b$ and $\omega_{ \pm}^{2}=v \pm i w$ one gets for the first two terms of Eq. (38) above the expression

$$
\begin{aligned}
\alpha_{+} f\left(p^{2}, \omega_{+}^{2}\right)+\alpha_{-} f\left(p^{2}, \omega_{-}^{2}\right)=\frac{1}{8 \pi}\{ & \frac{1}{p^{2}}\left[a \ell_{3}\left(p^{2}\right)+b a_{2}\left(p^{2}\right)\right] \\
& \left.+\frac{1}{R^{2}}\left[(a v+b w) \ell_{5}\left(p^{2}\right)-(b v-a w) a_{3}\left(p^{2}\right)\right]\right\},
\end{aligned}
$$

where

$$
\ell_{5}\left(p^{2}\right)=\ln \left(\frac{\sqrt{p^{4}+2 v p^{2}+R^{2}}}{p^{2}}\right)
$$

and $R, \ell_{3}\left(p^{2}\right), a_{2}\left(p^{2}\right)$ and $a_{3}\left(p^{2}\right)$ have already been defined in Eqs. (15), (30), (33) and (34). We also have

$$
i c \widetilde{f}\left(p^{2}, \omega_{+}^{2}, \eta\right)-i c \widetilde{f}\left(p^{2}, \omega_{-}^{2}, \eta\right)=-2 c \mathfrak{I}\left[\widetilde{f}\left(p^{2}, \omega_{+}^{2}, \eta\right)\right],
$$

where we have indicated with $\mathfrak{I}$ the imaginary part of the expression in square brackets.

\section{FITS TO GHOST-PROPAGATOR DATA}

The data for the ghost propagators $G\left(p^{2}\right)$ in $d=3,4$ and 2 have been evaluated for essentially the same set of lattice parameters considered for the gluon propagator $D\left(p^{2}\right)$ in Refs. $[1,18]$. A summary of the various lattice setups is presented in Table I. More details about the numerical simulations can be found in Ref. [1]. These simulations [12, 14, 15] have been done in 2007 using, in $3 d$ and in $4 d$, the 4.5 Tflops IBM supercomputer at LCCA-USP and, in the $2 d$ case, a PC cluster at the IFSC-USP. In all cases we set the lattice spacing $a$ by relating the lattice string tension $\sqrt{\sigma_{\text {latt }}}$ to the physical value $\sqrt{\sigma} \approx 0.44 \mathrm{GeV}$, which is a typical value for this quantity in the $4 d \mathrm{SU}(3)$ case. For $\sqrt{\sigma_{\text {latt }}}$ we used the results described in [74], [75] and [76], respectively for $d=3,4$ and 2. Note that all runs are in the scaling region and all data refer to the $\mathrm{SU}(2)$ case. Possible systematic effects due to Gribov copies [17, 77-82] or unquenching effects [83-87] were not considered.

Let us also recall that the Landau-gauge ghost propagator $G\left(p^{2}\right)$ is obtained by inverting the lattice Faddeev-Popov matrix $\mathcal{M}(b, x ; c, y)$ and is given by

$$
G^{b c}\left(p^{2}\right)=\sum_{x, y} \frac{e^{-2 \pi i \hat{p} \cdot(x-y) / N}}{V}\left\langle\mathcal{M}^{-1}(b, x ; c, y)\right\rangle=\delta^{b c} G\left(p^{2}\right),
$$

where $b$ and $c$ are color indices and \langle\rangle stands for the path-integral average. The inversion of the Faddeev-Popov matrix is obtained by using a conjugate gradient method with even/odd preconditioning and point sources [88, 89]. For the lattice Faddeev-Popov matrix we consider Eq. (22) in Ref. [90]. At the same time, the momentum components $p_{\mu}$ are given by

$$
p_{\mu}=2 \sin \left(\frac{\pi \hat{p}_{\mu}}{N}\right)
$$




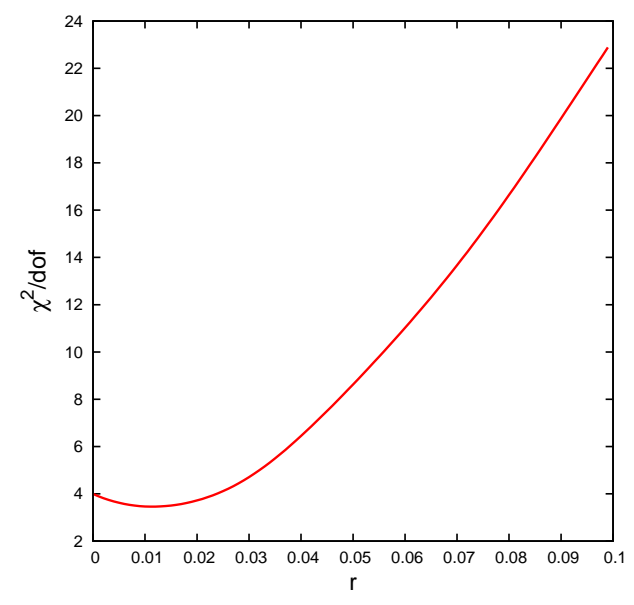

FIG. 1. Plot of the average reduced chi-squared statistic $\chi^{2} / d o f$ as a function of the parameter $r$ [see Eq. (47) and explanation in the text]. The average is taken over all lattice volumes $V$ and $\beta$ values considered.

and $\hat{p}_{\mu}$ takes the values $0,1, \ldots, N-1$. However, since the Faddeev-Popov matrix has a trivial null eigenvalue corresponding to a constant eigenvector, one cannot evaluate the ghost propagator at zero momentum, i.e. with $\hat{p}_{\mu}=0$ for all directions $\mu$. For the nonzero momenta, we considered in $2 d$ all momenta with components $(\bar{p}, 0)$ and $(\bar{p}, \bar{p})$, plus all possible permutations of the components. Similarly, in $3 d$ and in $4 d$ we present results for momenta of the type $(\bar{p}, 0,0),(\bar{p}, \bar{p}, 0),(\bar{p}, \bar{p}, \bar{p})$ and of the type $(\bar{p}, 0,0,0),(\bar{p}, \bar{p}, 0,0),(\bar{p}, \bar{p}, \bar{p}, 0)$ and $(\bar{p}, \bar{p}, \bar{p}, \bar{p})$, respectively. ${ }^{5}$

Finally, we recall that the best fits for the gluon propagator data, reported in Refs. [1, 18] and used here as theoretical inputs,

\begin{tabular}{|c|c|c|c|c|c|c|}
\hline$V=N^{d}$ & $\beta$ & $\#$ confs & $a($ fermi $)$ & $L=N a($ fermi $)$ & $p_{\min }(\mathrm{MeV})$ & $r$ \\
\hline \hline $140^{3}$ & 3.0 & 626 & 0.268 & 37.5 & 33.0 & 0.018 \\
\hline $200^{3}$ & 3.0 & 484 & 0.268 & 53.6 & 23.1 & 0.006 \\
\hline $240^{3}$ & 3.0 & 343 & 0.268 & 64.3 & 19.2 & 0.000 \\
\hline $320^{3}$ & 3.0 & 122 & 0.268 & 85.8 & 14.4 & 0.012 \\
\hline \hline $48^{4}$ & 2.2 & 99 & 0.210 & 10.1 & 122.7 & 0.017 \\
\hline $56^{4}$ & 2.2 & 100 & 0.210 & 11.8 & 105.2 & 0.007 \\
\hline $64^{4}$ & 2.2 & 100 & 0.210 & 13.4 & 92.1 & 0.047 \\
\hline $80^{4}$ & 2.2 & 97 & 0.210 & 16.8 & 73.7 & 0.021 \\
\hline $128^{4}$ & 2.2 & 21 & 0.210 & 26.9 & 46.0 & 0.012 \\
\hline \hline $80^{2}$ & 10.0 & 600 & 0.219 & 17.5 & 70.6 & 0.006 \\
\hline $120^{2}$ & 10.0 & 600 & 0.219 & 26.3 & 47.1 & 0.005 \\
\hline $160^{2}$ & 10.0 & 600 & 0.219 & 35.0 & 35.3 & 0.008 \\
\hline $200^{2}$ & 10.0 & 600 & 0.219 & 43.8 & 28.3 & 0.001 \\
\hline $240^{2}$ & 10.0 & 600 & 0.219 & 52.6 & 23.5 & 0.015 \\
\hline $280^{2}$ & 10.0 & 600 & 0.219 & 61.3 & 20.2 & 0.000 \\
\hline $320^{2}$ & 10.0 & 600 & 0.219 & 70.1 & 17.7 & 0.008 \\
\hline
\end{tabular}

TABLE I. For each lattice volume $V$ and lattice coupling $\beta$ we indicate the number of configurations considered, the value of the lattice spacing $a$ in fermi, the lattice size $L$ (also in fermi), the value of the smallest nonzero momentum $p_{\min }=2 \sin (\pi / N) / a$ (in MeV) and the coefficient $r$ that allows the largest reduction of the rotational-symmetry-breaking effects [see Eq. (47) and explanation in the text].

\footnotetext{
${ }^{5}$ Again, for each kinematic setup, we consider all possible permutations of the momentum components. When permutations of the momentum components were available, the average over different permutations was taken independently for each configuration.
} 

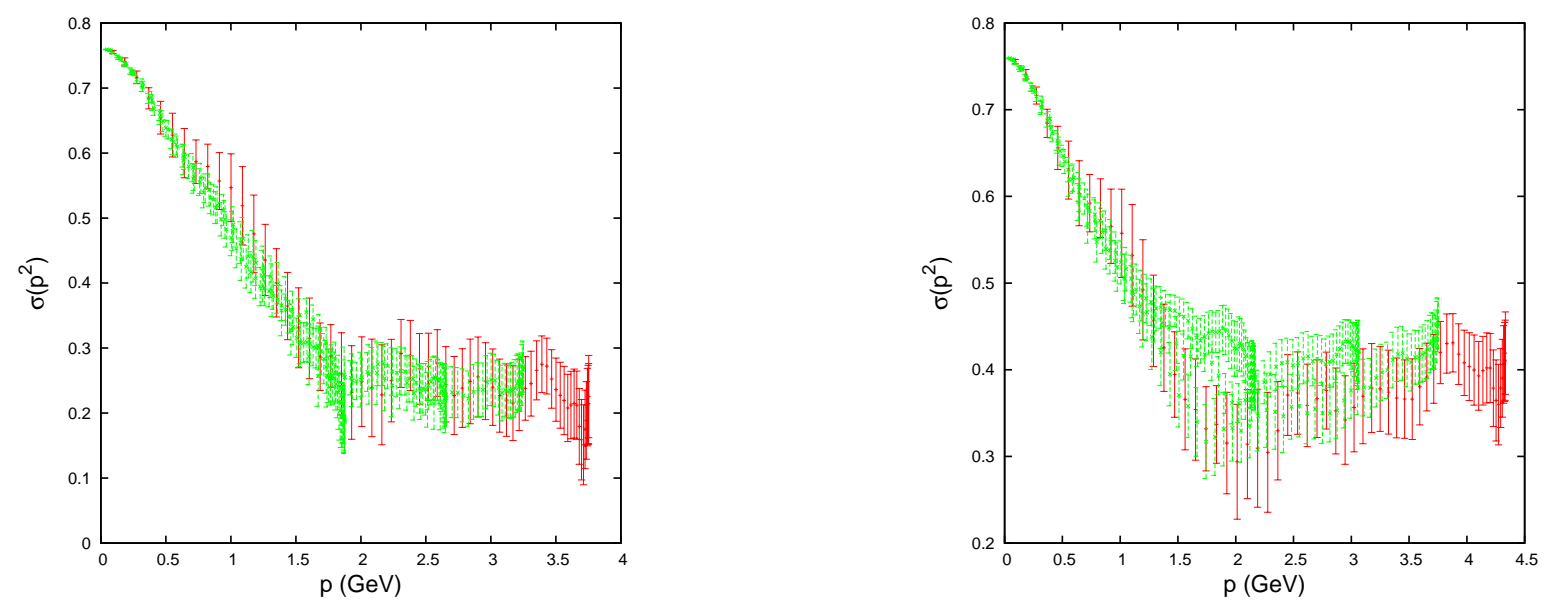

FIG. 2. Plot of the Gribov ghost form factor $\sigma\left(p^{2}\right)$ [see Eq. (2)] for the lattice volume $V=128^{4}$ at $\beta=2.2$, as a function of unimproved momenta [see Eq. (48)] (left plot) and of improved momenta [see Eq. (47)] with $r=1 / 12$ (right plot). In both plots, red data points correspond to momenta along the diagonal direction $\left(p_{\mu}=\bar{p}\right.$ for $\left.\mu=1, \ldots, 4\right)$, while green data points correspond to off-diagonal momenta. All momenta are in physical units. Error bars have been estimated using propagation of errors.

were obtained by considering this propagator as a function of the improved magnitude squared of the momentum [91]

$$
p^{2}=\sum_{\mu} p_{\mu}^{2}+r \sum_{\mu} p_{\mu}^{4}
$$

with $r=1 / 12 \approx 0.083$. This allows a better control of systematic effects - related to the breaking of rotational symmetry [91-93] — than the usual unimproved definition

$$
p^{2}=\sum_{\mu} p_{\mu}^{2}
$$

On the other hand, for the ghost propagator, the data are generally smoother when using the above unimproved definition, ${ }^{6}$ or a very small value of $r$. In order to verify this, we have considered the momentum behavior of the Gribov ghost form factor $\sigma\left(p^{2}\right)$ [defined in Eq. (2)] as a function of the improved magnitude squared of the momenta (47) for 100 different values of the parameter $r$, i.e. $r=0,0.001,0.002,0.003, \ldots, 0.099$. For each of these values, we used a cubic spline interpolation to obtain a description of the ghost-propagator data along the diagonal momentum direction, ${ }^{7}$ i.e. for $p_{\mu}=\bar{p}$ and $\mu=1, \ldots, d$. Then, we have evaluated the goodness of the fit, i.e. the reduced chi-squared statistic $\chi^{2} / d o f$, by comparing this interpolated curve with ghost-propagator data off the diagonal-momentum direction, i.e. with at least one momentum component equal to zero. In the last column of Table I we report, for each lattice volume $V$ and lattice coupling $\beta$, the value of the parameter $r$ that yields the smallest value for the reduced chi-squared statistic. As one can clearly see, these values of $r$ are very small for most of the cases considered. We also show in Fig. 1 the average value (over all lattice volumes $V$ and $\beta$ values considered) of $\chi^{2} /$ dof as a function of the parameter $r$. Again, we see that for small values of $r$ the $\chi^{2} /$ dof curve is almost flat, with a minimum value around $r=0.01$, and that for $r \geq 0.03$ the average value of the reduced chi-squared increases almost linearly. The effect of using a large value of the parameter $r$ can also be clearly visualized in the plots reported in Fig. 2, where we show the data for $\beta=2.2$ and our largest $4 d$ lattice $V=128^{4}$ as a function of unimproved momenta (left plot) and as a function of "improved" momenta ${ }^{8}$ with $r=1 / 12$ (right plot). Indeed, the spread of the data points is clearly larger in the second case. Thus, for simplicity's sake, we will consider below all the ghost-propagator data as a function of the unimproved momenta [see Eq. (48)]. One should, of course, try to reduce discretization effects in order to obtain results closer to the continuum formulation of the theory, but we

\footnotetext{
${ }^{6}$ This is probably related to the fact that the ghost propagator $G\left(p^{2}\right)$ [see Eq. (45)] does not depend explicitly on the Lorentz index $\mu$.

7 This direction is usually less affected by rotational-symmetry-breaking effects [92, 93].

${ }^{8}$ This value of $r$ is usually employed in fits of the gluon propagator (see e.g. [1]).
} 


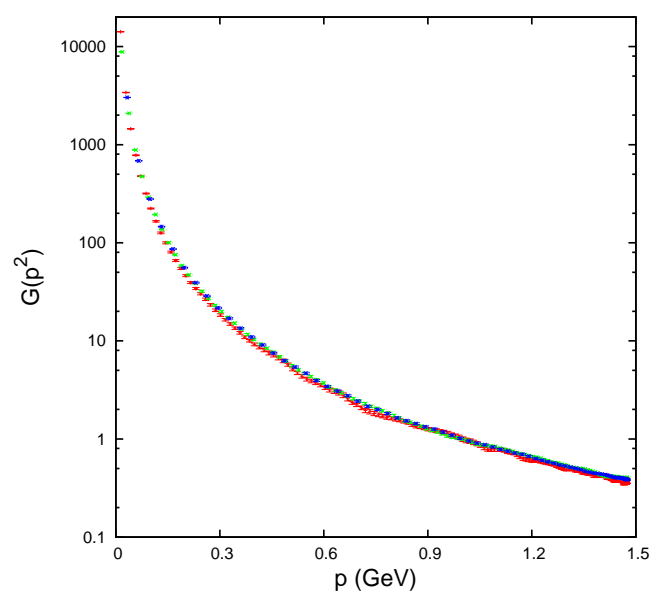

FIG. 3. Plot of the ghost propagator $G\left(p^{2}\right)$ as a function of the magnitude of the (unimproved) momenta $p$ (both in physical units) for the lattice volumes $V=140^{3}$ (symbol $*$ in blue), $V=240^{3}$ (symbol $\times$ in green) and $V=320^{3}$ (symbol + in red) at $\beta=3.0$. Here we show the data corresponding to momenta with only one component different from zero. The data are (multiplicatively) normalized to 1 for $p=1.0 \mathrm{GeV}$. Notice the logarithmic scale on the $y$ axis.

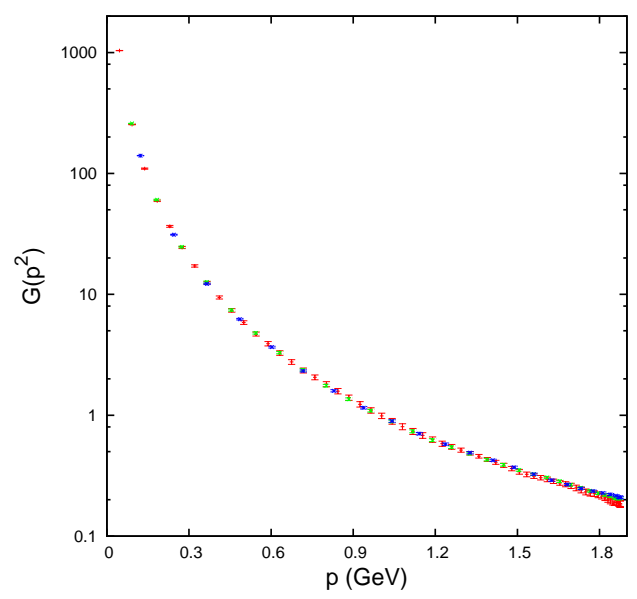

FIG. 4. Plot of the ghost propagator $G\left(p^{2}\right)$ as a function of the magnitude of the (unimproved) momenta $p$ (both in physical units) for the lattice volumes $V=48^{4}$ (symbol $*$ in blue), $V=64^{4}$ (symbol $\times$ in green) and $V=128^{4}$ (symbol + in red) at $\beta=2.2$. Here we show the data corresponding to momenta with only one component different from zero. The data are (multiplicatively) normalized to 1 for $p=1.0 \mathrm{GeV}$. Notice the logarithmic scale on the $y$ axis.

must note that different lattice quantities are subject in general to different such effects. Thus, it is not surprising that gluon and ghost propagator data require different definitions of the lattice momenta when one tries to connect lattice data to the continuum analysis carried out in Ref. [64].

In the next three subsections we present the modeling of the numerical data for $G\left(p^{2}\right)$. In analogy with the presentation of the one-loop calculations in Section II, we first give our results for the $3 d$ case, then for the $4 d$ case and, finally, for the $2 d$ case. Let us note that finite-size effects for $G\left(p^{2}\right)$ are generally negligible. This can be seen in Figs. 3, 4 and 5, where $G\left(p^{2}\right)$ is plotted for three different lattice sizes respectively for $d=3,4$ and 2 . We also remark that the use of the point-source method in the evaluation of $G\left(p^{2}\right)$ leads to the slight "wiggling" of the lattice data seen in the three plots above (see Ref. [89]). Thus, in the following, we will always use the largest lattice volume available for each dimension $d$. Also, in all cases we will show the data (multiplicatively) normalized to $1 / \bar{\mu}^{2}$ for $p=\bar{\mu}=2.5 \mathrm{GeV}$.

The analytic expression proposed for the ghost propagator will be cast in the form of Eq. (1), using in each dimension $d$ the 


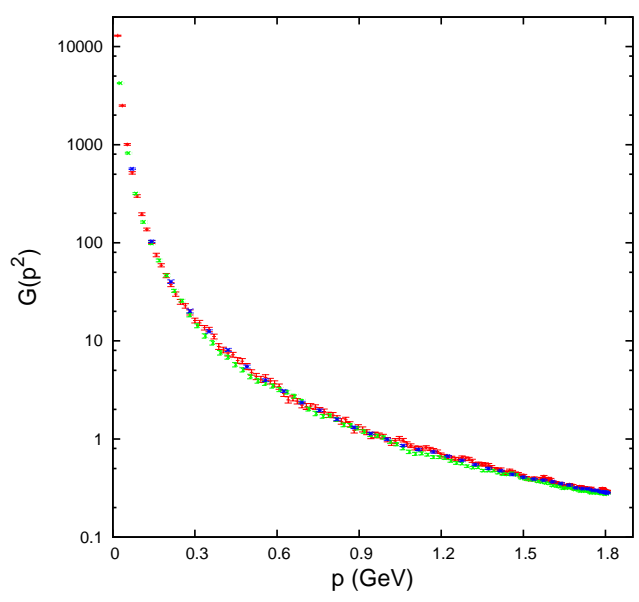

FIG. 5. Plot of the ghost propagator $G\left(p^{2}\right)$ as a function of the magnitude of the (unimproved) momenta $p$ (both in physical units) for the lattice volumes $V=80^{2}$ (symbol $*$ in blue), $V=200^{2}$ (symbol $\times$ in green) and $V=320^{2}$ (symbol + in red) at $\beta=10.0$. Here we show the data corresponding to momenta with only one component different from zero. The data are (multiplicatively) normalized to 1 for $p=1.0 \mathrm{GeV}$. Notice the logarithmic scale on the $y$ axis.

corresponding one-loop results [64] for the Gribov ghost form factor $\sigma\left(p^{2}\right)$ listed in Section II above. The parameters in $\sigma\left(p^{2}\right)$ will be taken from the gluon-propagator results obtained in Refs. $[1,18]$. Then, the only parameter left is the bare coupling constant $g^{2}$. As explained below, $g^{2}$ is set in the $3 d$ and $2 d$ cases by considering its relation to the string tension $\sqrt{\sigma}$, while in the $4 d$ case we adopt the value of $g^{2}\left(\bar{\mu}^{2}\right)$ at the scale $\bar{\mu}$ in the MOM scheme.

In order to normalize the analytic expressions for $G\left(p^{2}\right)$ consistently with the lattice data, we consider two possibilities. In the first case, we take

$$
F_{1}\left(p^{2}\right)=\frac{1-\sigma\left(\bar{\mu}^{2}\right)}{p^{2}\left[1-\sigma\left(p^{2}\right)\right]}
$$

Alternatively, as already discussed above in Section II B, one can normalize $G\left(p^{2}\right)$ by adding a constant to $\sigma\left(p^{2}\right)$, i.e. considering

$$
F_{2}\left(p^{2}\right)=\frac{1}{p^{2}\left[1-\sigma\left(p^{2}\right)+\sigma\left(\bar{\mu}^{2}\right)\right]} .
$$

Let us stress that, with the parameters fixed as above, these functions are not fitting forms, but analytic predictions for $G\left(p^{2}\right)$ from previously obtained (gluon-propagator) results. These will allow a good description of the lattice data in the ultraviolet (UV) regime only. Nevertheless, by treating $g^{2}$ as a free parameter in the above formulae and keeping the remaining parameters fixed, one obtains good-quality fits for the whole range of data in all cases. We indicate the corresponding fitting forms by $\widetilde{F}_{1}\left(p^{2}\right)$ and $\widetilde{F}_{2}\left(p^{2}\right)$.

\section{A. The three-dimensional case}

As discussed above, we now try to describe the ghost-propagator data in $d=3$ by considering the Gribov ghost form factor $\sigma\left(p^{2}\right)$ given by $\sigma_{1 L}\left(p^{2}\right)$ [see Eqs. (12)-(22)]. We set the parameters $\alpha, \omega_{1}, a, b, v, w$ to the values obtained in Refs. [1, 18] by fitting the gluon propagator. In particular, we use the values reported in Table XI of Ref. [1] (from a Monte Carlo analysis), i.e.

$$
\begin{gathered}
a=0.216(2) \mathrm{GeV}, \quad b=0.271(3) \mathrm{GeV}, \quad v=0.215(5) \mathrm{GeV}^{2}, \\
w=0.580(6) \mathrm{GeV}^{2}, \quad \alpha=-0.024(5) \mathrm{GeV}, \quad \omega_{1}^{2}=0.046(4) \mathrm{GeV}^{2} .
\end{gathered}
$$


As for the bare coupling $g^{2}$, since in $3 d$ it is a constant (mass) parameter, ${ }^{9}$ we use the $\mathrm{SU}(2)$ ratio $\sqrt{\sigma} / g^{2}=0.3351(16)$ [see Eq. (7) of Ref. [94]]. Then, with $\sqrt{\sigma} \approx 0.44 \mathrm{GeV}$ we find $g^{2} \approx 1.313 \mathrm{GeV}$. The corresponding plot of $F_{1}\left(p^{2}\right)$ (see above) is shown in Fig. 6 (left plot). Let us point out that for the momentum range spanned by the data the functions $F_{1}\left(p^{2}\right)$ and $F_{2}\left(p^{2}\right)$ are numerically indistinguishable (see Fig. 7). Note that $\sigma_{1 L}\left(\bar{\mu}^{2}\right) \approx 0.0252$ and that $\sigma_{1 L}\left(p^{2}\right)$ takes values ${ }^{10}$ in $[0.0247,0.1014]$ when $p \in[0.014,2.553] \mathrm{GeV}$. Let us also mention that the one-loop expression $p^{2} F_{1}\left(p^{2}\right)$ does not change appreciably in the considered momentum range. (It goes from about 1.0 in the UV to about 1.1 in the IR regimes.) Thus, the momentum dependence of the analytic prediction $F_{1}\left(p^{2}\right)$ is almost entirely due to the factor $1 / p^{2}$.

One can observe that, modulo a global factor, $F_{1}\left(p^{2}\right)$ has the expected leading UV and IR behaviors. Indeed, as shown in Fig. 6 (right plot), it agrees with the data in the IR limit if we consider $3.38353 F_{1}\left(p^{2}\right)$. This implies that, in the deep IR limit, $G\left(p^{2}\right)$ is enhanced by a finite multiplicative factor with respect to the UV behavior. As mentioned above, one can improve the description of the ghost-propagator data in the whole momentum range by fitting the values of $g^{2}$, instead of using a fixed value. In this case, we find

$$
g^{2}=10.08 \pm 0.01 \mathrm{GeV}
$$

with $\chi^{2} / d o f \approx 4.5$ (with 480 data points). The corresponding plot of $\widetilde{F}_{1}\left(p^{2}\right)$ is shown in Fig. 8 (left plot). Let us stress that, with this fitted value for $g^{2}$, the analytic prediction $p^{2} \widetilde{F}_{1}\left(p^{2}\right)$ varies from about 1.0 at large momentum to about 3.6 in the IR limit, a behavior that can be related to the global rescaling shown in Fig. 6 . An even better fit of the data can be obtained with the fitting function [15]

$$
F_{3}\left(p^{2}\right)=\frac{z}{p^{2}} \frac{t+p^{2} / s^{2}+\log \left(1+p^{2} / s^{2}\right)}{1+p^{2} / s^{2}},
$$

inspired by Ref. [27], which has $1 / p^{2}$ leading IR and UV behaviors. Indeed, with the fitting parameters set to

$$
\begin{aligned}
& z=0.958 \pm 0.004 \\
& t=3.81 \pm 0.02 \\
& s=0.207 \pm 0.003 \mathrm{GeV}
\end{aligned}
$$

we find a $\chi^{2} / d o f \approx 2.9$ (again with 480 data points). The corresponding plot is shown in Fig. 8 (right plot). Note that the value of the parameter $t$ is compatible with the multiplicative constant obtained above when comparing the IR and UV behaviors of $F_{1}\left(p^{2}\right)$ (see Fig. 6).

One can try to estimate what is missing in the RGZ one-loop analysis for $G\left(p^{2}\right)$, e.g. using the expression for $F_{2}\left(p^{2}\right)$ in Eq. (50). More precisely, let us define a function $W\left(p^{2}\right)$ by

$$
G\left(p^{2}\right)=\frac{1}{p^{2}\left[1-\sigma\left(p^{2}\right)+\sigma\left(\bar{\mu}^{2}\right)-W\left(p^{2}\right)\right]}
$$

and then use our numerical data for $G\left(p^{2}\right)$ [and the one-loop expression for $\left.\sigma\left(p^{2}\right)\right]$ to get an estimate for $W\left(p^{2}\right)$. To this end, we carried out a Monte Carlo analysis (with 10000 samples) of the quantity

$$
W\left(p^{2}\right)=\left[1-\frac{1}{p^{2} G\left(p^{2}\right)}\right]-\sigma_{1 L}\left(p^{2}\right)+\sigma_{1 L}\left(\bar{\mu}^{2}\right),
$$

where $G\left(p^{2}\right)$ represents the numerical (multiplicatively normalized) ghost propagator result at a given momentum $p$ and $\sigma_{1 L}\left(p^{2}\right)$ is the one-loop estimate (12)-(22) with the parameters given in Eq. (51) and the value of $g^{2}$ set to $1.313 \mathrm{GeV}$. The corresponding plot is shown in Fig. 9. The estimated error for $W\left(p^{2}\right)$ includes the error in the data points for $G\left(p^{2}\right)$ and the errors in the

\footnotetext{
${ }^{9}$ Let us recall that, in the general $d$-dimensional case, we have that $g^{2}$ has mass dimension $4-d$.

${ }^{10}$ As shown in Ref. [64], from Eq. (9) one can write

$$
\sigma\left(p^{2}\right)=\frac{N_{c} p_{\mu} p_{v}}{p^{2}} \int \frac{\mathrm{d}^{d} q}{(2 \pi)^{d}} D\left(q^{2}\right) P_{\mu v}(q) \frac{1}{(p-q)^{2}}
$$
}

and prove that $\partial \sigma\left(p^{2}\right) / \partial p^{2}<0$ if the gluon propagator $D\left(p^{2}\right)$ is positive in momentum space, i.e. $\sigma\left(p^{2}\right)$ —evaluated at one loop- is monotonically decreasing as the momentum $p$ increases. 

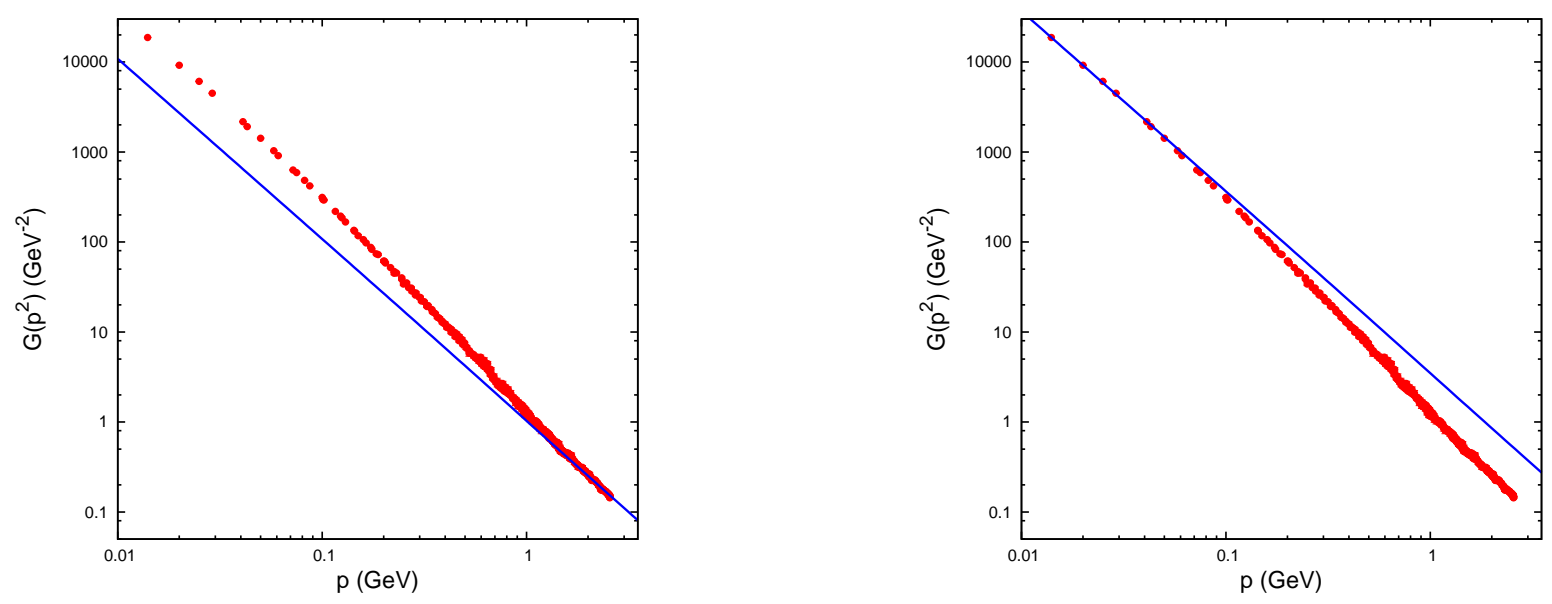

FIG. 6. Plot of the ghost propagator $G\left(p^{2}\right)$ as a function of the magnitude of the (unimproved) momenta $p$ (both in physical units) for the lattice volume $V=320^{3}$ at $\beta=3.0$. The data are (multiplicatively) normalized to $1 / \bar{\mu}^{2}$ for $p=\bar{\mu}=2.5 \mathrm{GeV}$. We also show the function $F_{1}\left(p^{2}\right)$ [see Eq. (49)] (normalized in the same way) with the Gribov ghost form factor $\sigma\left(p^{2}\right)$ given by the one-loop results (12)-(22); the corresponding parameters are reported in Eq. (51) and we set $g^{2}=1.313 \mathrm{GeV}$. On the other hand, in the right plot, we fix the analytic form to match the numerical result at $p=p_{\min }=14 \mathrm{MeV}$, the smallest nonzero (lattice) momentum for the pair $(V, \beta)$ considered, yielding $3.38353 F_{1}\left(p^{2}\right)$. Notice the logarithmic scale on both axes.

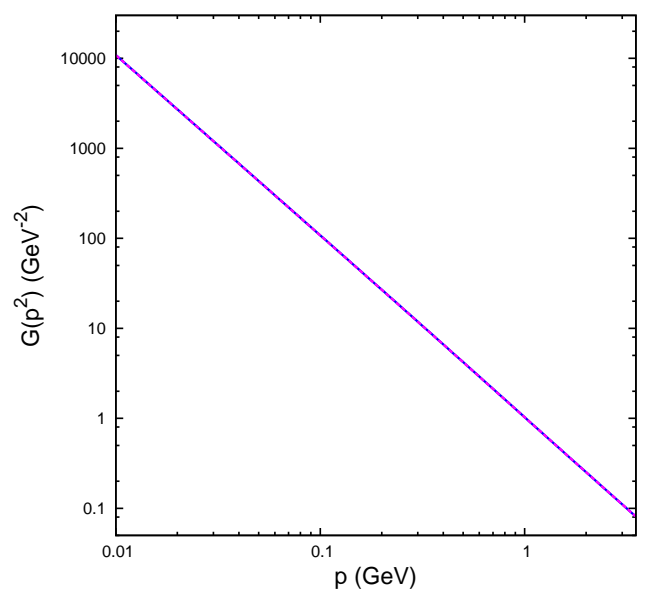

FIG. 7. Plot of $F_{1}\left(p^{2}\right)$ [see Eq. (49), blue curve] and $F_{2}\left(p^{2}\right)$ [see Eq. (50), magenta curve] as functions of the momentum $p$ for the $3 d$ case, with $\sigma\left(p^{2}\right)$ given by $\sigma_{1 L}\left(p^{2}\right)$ [see Eqs. (12)-(22) and (51)] with $g^{2}=1.313 \mathrm{GeV}$. For both curves we consider $\bar{\mu}=2.5 \mathrm{GeV}$. Notice the logarithmic scale on both axes.

parameters. Note that $W\left(p^{2}\right)$ goes from approximately zero in the UV regime to about 0.7 in the IR limit, which is consistent with the small variation of $p^{2} F_{1}\left(p^{2}\right)$ discussed above.

One can parametrize the function $W\left(p^{2}\right)$ reasonably well by using the simple expression

$$
\widetilde{W}\left(p^{2}\right)=\frac{A}{1+B p^{2}}
$$

with

$$
A \approx 0.64, \quad B \approx 3.4 \mathrm{GeV}^{-2},
$$

yielding a $\chi^{2} /$ dof of 2.7 (with 480 data points). The corresponding plot (red curve) is also shown in Fig. 9. 

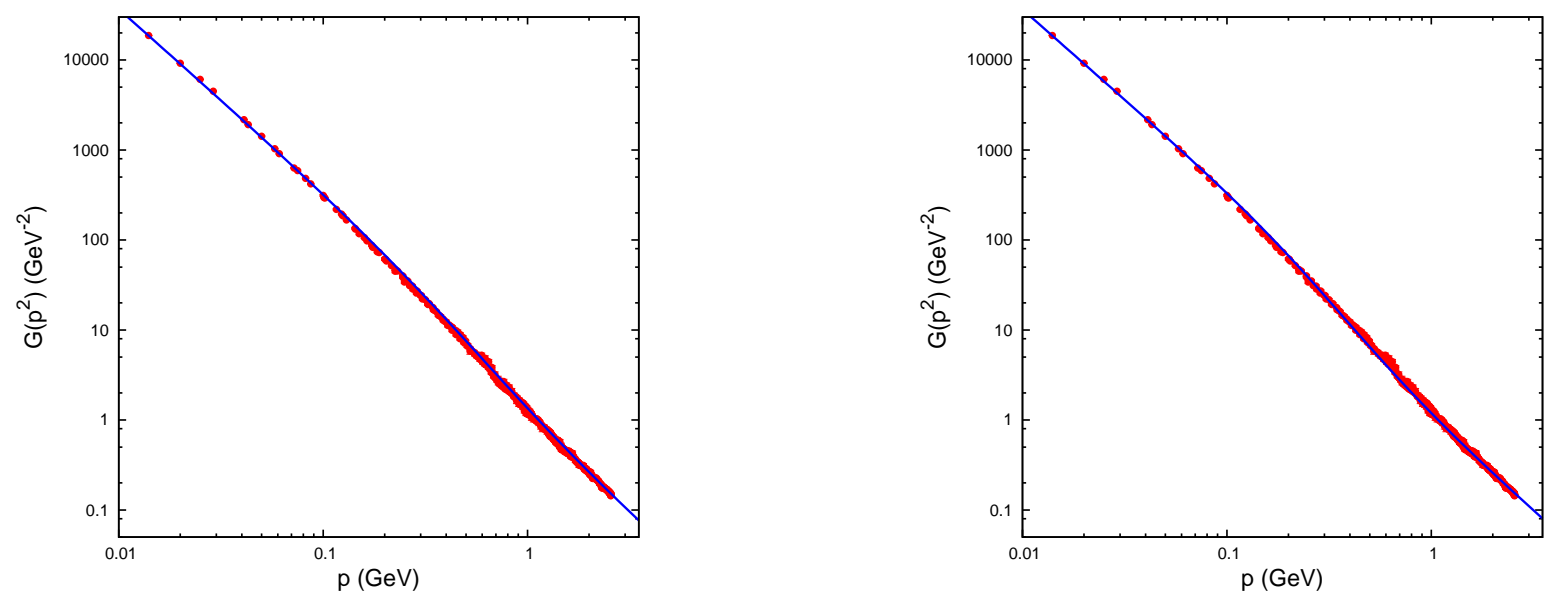

FIG. 8. Plot of the ghost propagator $G\left(p^{2}\right)$ as a function of the magnitude of the (unimproved) momenta $p$ (both in physical units) for the lattice volume $V=320^{3}$ at $\beta=3.0$ together with the fitting forms discussed in the text. The data are (multiplicatively) normalized to $1 / \bar{\mu}^{2}$ for $p=\bar{\mu}=2.5 \mathrm{GeV}$. In the left plot we show the function $\widetilde{F}_{1}\left(p^{2}\right)$ (normalized in the same way) with the Gribov ghost form factor $\sigma\left(p^{2}\right)$ given by the one-loop results (12)-(22); the corresponding parameters are reported in Eq. (51) and we use the fitted value $10.0831 \mathrm{GeV}$ for $g^{2}$. On the other hand, in the right plot, we show the fitting function $F_{3}\left(p^{2}\right)$ [see Eq. (54)] with the parameters given in Eqs. (55)-(57). Notice the logarithmic scale on both axes.

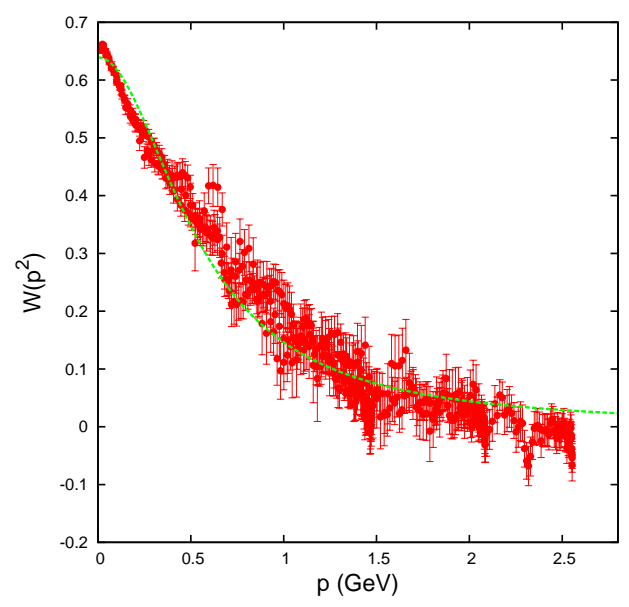

FIG. 9. Plot of the term $W\left(p^{2}\right)$ [see Eqs. (58) and (59)] as a function of the (unimproved) momenta $p$ (in physical units) for the lattice volume $V=320^{3}$ at $\beta=3.0$ (data points). We also show (in red) the fitting function $\widetilde{W}\left(p^{2}\right)$ [see Eq. (60)] with the fitting parameters reported in Eq. (61).

\section{B. The four-dimensional case}

For the $4 d$ case we repeat the same type of analysis carried out in the previous section for the $3 d$ case. In particular, as explained in Section II B above, we consider the function $F_{2}\left(p^{2}\right)$ in Eq. (50) with the one-loop expression for $\sigma\left(p^{2}\right)$ given by $\sigma_{1 L}^{\overline{M S}}\left(p^{2}\right)$ [see Eqs. (23)-(34)] and $\bar{\mu}=2.5 \mathrm{GeV}$. Again, by using the (gluon-propagator) results presented in Refs. [1, 18], the parameters $a, b, v, w$ are set to the values reported in Table IV of Ref. [1] and obtained using a Monte Carlo analysis, i.e.

$$
a=0.392(2), \quad b=1.32(5), \quad v=0.29(2) \mathrm{GeV}^{2}, \quad w=0.66(1) \mathrm{GeV}^{2} .
$$



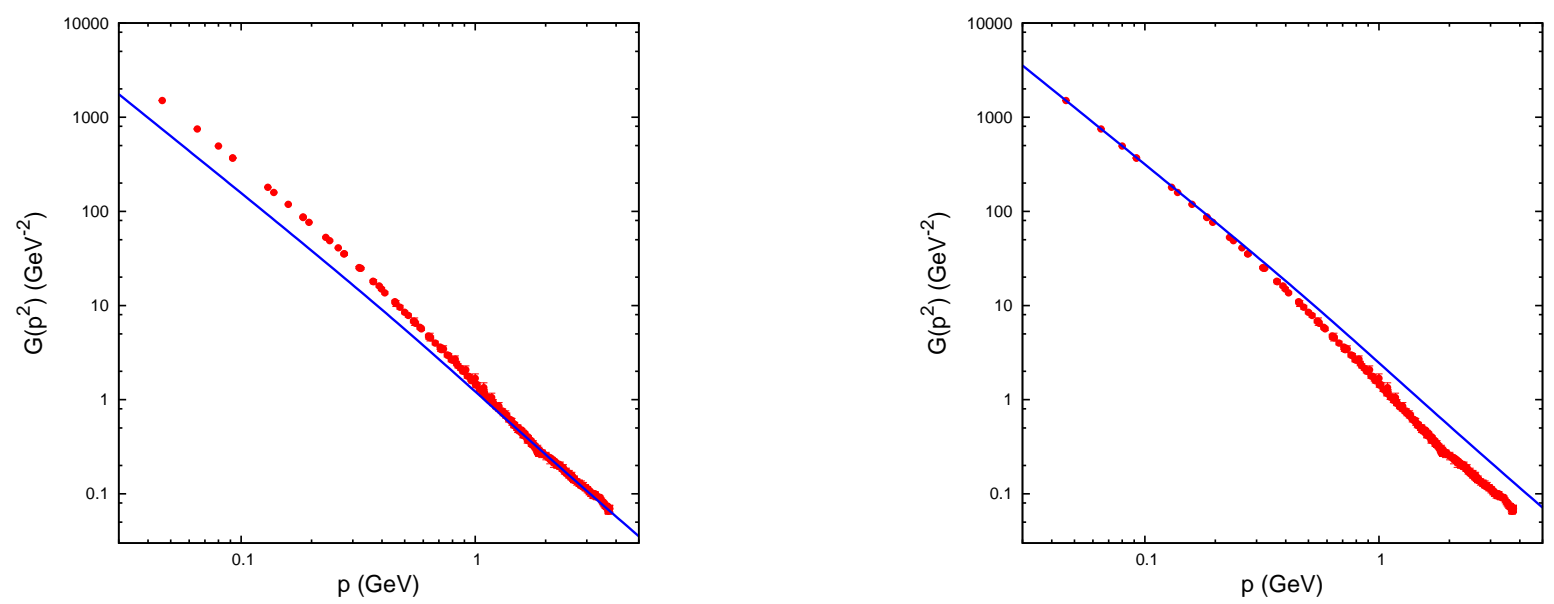

FIG. 10. Plot of the ghost propagator $G\left(p^{2}\right)$ as a function of the magnitude of the (unimproved) momenta $p$ (both in physical units) for the lattice volume $V=128^{4}$ at $\beta=2.2$. The data are (multiplicatively) normalized to $1 / \bar{\mu}^{2}$ for $p=\bar{\mu}=2.5 \mathrm{GeV}$. We also show the function $F_{2}\left(p^{2}\right)$ [see Eq. (50)] (normalized in the same way) with the Gribov ghost form factor $\sigma\left(p^{2}\right)$ given by the one-loop results (23)-(34); the corresponding parameters are reported in Eq. (62) and we set $g^{2}=7.794$. On the other hand, in the right plot, we fix the analytic form to match the numerical result at $p=p_{\text {min }}=46 \mathrm{MeV}$, the smallest nonzero (lattice) momentum for the pair $(V, \beta)$ considered, yielding $2.01654 F_{2}\left(p^{2}\right)$. Notice the logarithmic scale on both axes.

Here we can estimate the value of $g^{2}$, at a given scale $\bar{\mu}$ and in the MOM scheme, by considering the one-loop result

$$
g^{2}(\bar{\mu})=\frac{1}{\beta_{0} \ln \left(\frac{\vec{\mu}^{2}}{\Lambda_{\mathrm{MOM}}^{2}}\right)}
$$

with $\beta_{0}=11 N_{c} /\left(48 \pi^{2}\right)$, which is valid for any $\mathrm{SU}\left(N_{c}\right)$ gauge group. Then, the value of $\Lambda_{\text {мом }}$ can be obtained by considering the relation [95]

$$
g^{2}=\bar{g}^{2}\left(1+\frac{169 N_{c}}{36} \bar{g}^{2}+\ldots\right)
$$

between the MOM-scheme coupling $g^{2}$ and the $\overline{\mathrm{MS}}$ coupling $\bar{g}^{2}$. This implies (see for example [96]) $\Lambda_{\text {мом }}=\Lambda_{\overline{\mathrm{Ms}}} e^{169 / 264}$, which is valid for any value of $N_{c}$ and with $N_{f}=0$, where $N_{f}$ is the number of quark flavors. For the SU(2) case, i.e. for $N_{c}=2$, one can use the estimate $\Lambda_{\overline{\mathrm{MS}}} \approx 0.752 \sqrt{\sigma}$ (see Ref. [97]), where $\sqrt{\sigma}$ is the string tension. Then, after setting $\sqrt{\sigma} \approx 0.44 \mathrm{GeV}$ we find $\Lambda_{\overline{\mathrm{MS}}} \approx 331 \mathrm{MeV}$ and $\Lambda_{\text {мом }} \approx 628 \mathrm{MeV}$. For the subtraction point $\bar{\mu}=2.5 \mathrm{GeV}$, used here, this gives for the effective MOM coupling a value of $g^{2}(\bar{\mu}) \approx 7.794$, which yields $\alpha_{s}(\bar{\mu})=g^{2}(\bar{\mu}) /(4 \pi) \approx 0.6202$. The corresponding plot of $F_{2}\left(p^{2}\right)$ is shown in Fig. 10 (left plot). Also in this case, the functions $F_{1}\left(p^{2}\right)$ in Eq. (49) and $F_{2}\left(p^{2}\right)$ in Eq. (50) are numerically indistinguishable. Note that $\sigma_{1 L}^{\overline{\mathrm{MS}}}\left(\bar{\mu}^{2}\right) \approx 0.1419$ and that $\sigma_{1 L}^{\overline{\mathrm{MS}}}\left(p^{2}\right)$ takes values in $[0.06502,0.5081]$ when $p \in[0.046,3.752] \mathrm{GeV}$, which is the momentum interval for which we have numerically evaluated the ghost propagator $G\left(p^{2}\right)$. Here, contrary to the $3 d$ case, the one-loop expression $p^{2} F_{2}\left(p^{2}\right)$ is not flat with the momentum $p$, i.e. it changes from about 0.9 in the UV to about 1.6 in the IR regimes.

Qualitatively, the situation in the $4 d$ case is very similar to what we have seen above in the $3 d$ case. In particular, one can obtain a good description of the numerical data in the IR limit by rescaling the analytic prediction $F_{2}\left(p^{2}\right)$ by the factor 2.01654 (see right plot of Fig. 10) and a good description of all the data by fitting the values of $g^{2}$. Indeed, with

$$
g^{2}=14.62 \pm 0.01
$$

we obtain a $\chi^{2} / d o f \approx 1.7$ (with 256 data points). The corresponding plot of $\widetilde{F}_{2}\left(p^{2}\right)$ is shown in Fig. 11 (left plot). An even better fit (see right plot in Fig. 11) is obtained with the fitting function (54) and the parameters set to

$$
\begin{aligned}
& z=0.859 \pm 0.006 \\
& t=3.73 \pm 0.02 \\
& s=0.407 \pm 0.005 \mathrm{GeV},
\end{aligned}
$$



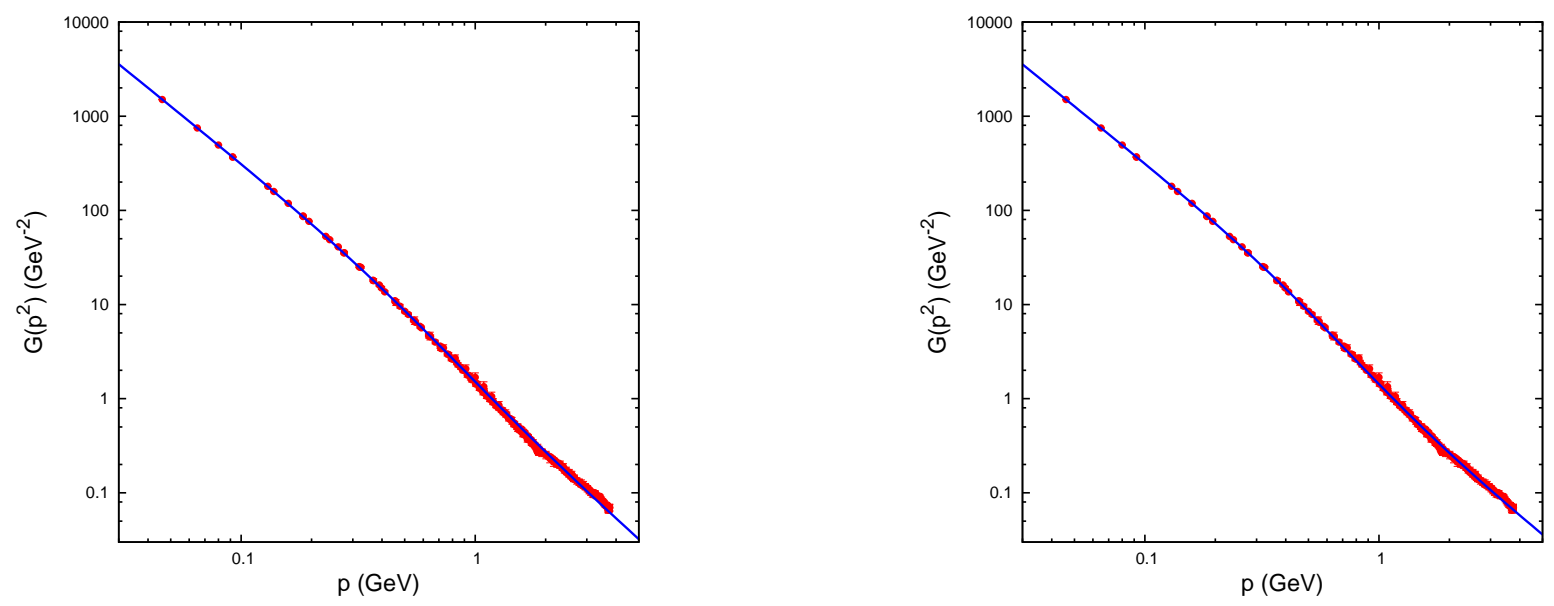

FIG. 11. Plot of the ghost propagator $G\left(p^{2}\right)$ as a function of the magnitude of the (unimproved) momenta $p$ (both in physical units) for the lattice volume $V=128^{4}$ at $\beta=2.2$ together with the fitting forms discussed in the text. The data are (multiplicatively) normalized to $1 / \bar{\mu}^{2}$ for $p=\bar{\mu}=2.5 \mathrm{GeV}$. In the left plot we show the function $\widetilde{F}_{2}\left(p^{2}\right)$ (normalized in the same way) with the Gribov ghost form factor $\sigma\left(p^{2}\right)$ given by the one-loop results (23)-(34); the corresponding parameters are reported in Eq. (62) and we use the fitted value 14.6165 for $g^{2}$. On the other hand, in the right plot, we show the fitting function $F_{3}\left(p^{2}\right)$ [see Eq. (54)] with the parameters given in Eqs. (66)-(68). Notice the logarithmic scale on both axes.

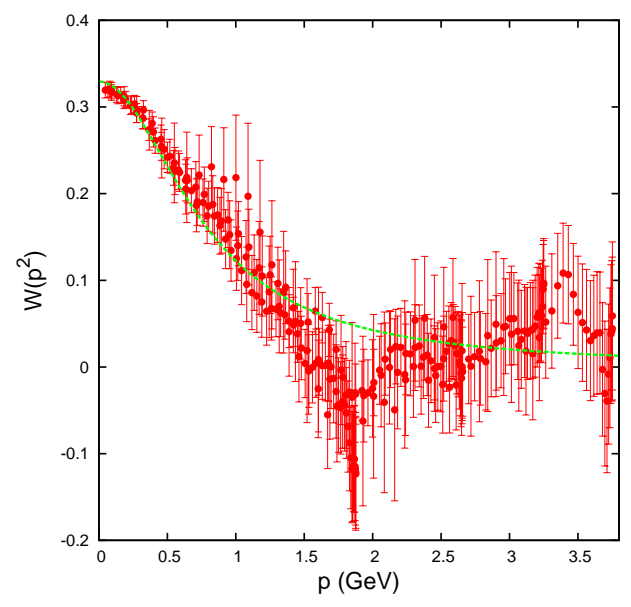

FIG. 12. Plot of the term $W\left(p^{2}\right)$ [see Eqs. (58) and (59)] as a function of the (unimproved) momenta $p$ (in physical units) for the lattice volume $V=128^{4}$ at $\beta=2.2$ (data points). We also show (in red) the fitting function $\widetilde{W}\left(p^{2}\right)$ [see Eq. (60)] with the fitting parameters reported in Eq. (69).

which yields a $\chi^{2} / d o f \approx 0.75$ (again with 256 data points). Here the value of $t$ can be related to the global rescaling shown on the right in Fig. 10 (i.e. approximately a factor 2 ) and to the above mentioned change in $p^{2} F_{2}\left(p^{2}\right)$, yielding a factor $2 \times 1.6 / 0.9 \approx 3.6$. The parameter $t$ can also be related to the variation of $p^{2} \widetilde{F}_{2}\left(p^{2}\right)$ from about 0.9 at large momentum to about 3.2 in the IR limit, yielding a factor $3.2 / 0.9 \approx 3.6$.

Finally, in Fig. 12 we present the numerical estimate —using a Monte Carlo analysis with 10000 samples — for the quantity $W\left(p^{2}\right)$, defined in Eq. (59) and using the $4 d$ one-loop expression for $\sigma\left(p^{2}\right)$, as well as the fitting function $\widetilde{W}\left(p^{2}\right)$, defined in Eq. (60). With the values

$$
A \approx 0.33, \quad B \approx 1.7 \mathrm{GeV}^{-2}
$$

for the parameters we find a $\chi^{2} / d o f$ of 0.97 (with 256 data points). It is also interesting to note that, in this case, the magnitude 


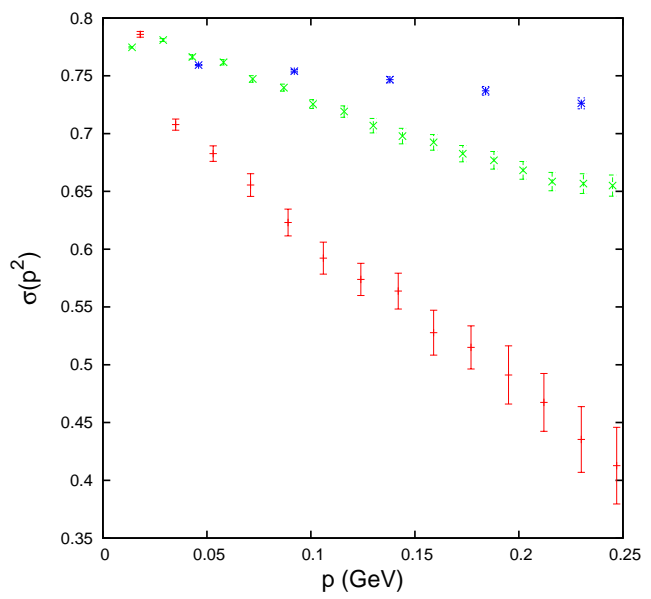

FIG. 13. Plot of the Gribov ghost form factor $\sigma\left(p^{2}\right)$ [see Eq. (2)] for the lattice volumes $V=320^{2}$ at $\beta=10.0$ (symbol + in red), $V=320^{3}$ at $\beta=3.0$ (symbol $\times$ in green) and $V=128^{4}$ at $\beta=2.2$ (symbol $*$ in blue), as a function of the unimproved momenta [see Eq. (48)], for momenta with only one component different from zero. All momenta are in physical units and we show the data only in the IR limit, i.e. for $p \leq 0.25 \mathrm{GeV}$. Error bars have been estimated using propagation of errors. One clearly sees that in the $3 d$ and $4 d$ cases $\sigma\left(p^{2}\right)$ becomes almost constant at small momenta, with a value $\sigma(0)<1$, implying a free-like behavior for the ghost propagator in the IR limit. On the contrary, in the $2 d$ case $\sigma\left(p^{2}\right)$ is still clearly increasing for momenta of the order of $20 \mathrm{MeV}$.

of what is missing in the one-loop calculation of $\sigma\left(p^{2}\right)$ is about $50 \%$ smaller than the corresponding outcome obtained in the $3 d$ case. This is expected since, as mentioned above, there is a larger change in $p^{2} F_{2}\left(p^{2}\right)$ over the momentum range in the $4 d$ case.

\section{The two-dimensional case}

Finally, we consider data for the $2 d$ case. As already stressed in the Introduction, in this case the ghost propagator is IRenhanced (see also Fig. 13). Thus, the analysis of the numerical data will be done following the same ideas presented in the two subsections above, but with a different fitting function instead of $F_{3}\left(p^{2}\right)$ in Eq. (54). Nevertheless, as a first step, we consider again the one-loop result $F_{1}\left(p^{2}\right)$ [see Eq. (49)] with $\sigma\left(p^{2}\right)$ given by $\sigma_{2 d}\left(p^{2}\right)$ defined in Eqs. (38)-(44) and with $\bar{\mu}=2.5 \mathrm{GeV}$. At the same time, the parameters $a, b, v, w, c$ and $\eta$ are set considering the outcomes presented in Refs. [1, 18]. In particular, we used the values reported in Table XIV of Ref. [1] and obtained using a Monte Carlo analysis, i.e.

$$
\begin{gathered}
a=0.0550(5) \mathrm{GeV}^{2}, \quad b=-0.049(7) \mathrm{GeV}^{2}, \quad v=0.145(8) \mathrm{GeV}^{2}, \\
w=0.15(1) \mathrm{GeV}^{2}, \quad c=0.07(1) \mathrm{GeV}^{2-\eta}, \quad \eta=0.91(5) .
\end{gathered}
$$

Note that the bound $\eta<2$ (see Sec. IIC) is respected by the fitted value. As for the he coupling constant $g^{2}$, its value can be estimated by employing the analytic evaluation of the string tension $\sqrt{\sigma}$. Indeed, for two-dimensional $\mathrm{SU}(2)$ lattice gauge theory in the infinite volume limit, one has [98]

$$
\sigma_{\text {latt }}=-\ln \left[\frac{I_{2}(\beta)}{I_{1}(\beta)}\right]
$$

where $I_{n}(\beta)$ is the modified Bessel function [73]. For large $\beta$ values (in our case we have $\beta=10$ ), this yields $\sigma_{\text {latt }} \approx 3 /(2 \beta)$. Then, using the relation $\beta=2 N_{c} /\left(g^{2} a^{4-d}\right)$, where $a$ is the lattice spacing and which is valid for the $\mathrm{SU}\left(N_{c}\right)$ gauge group in $d$ dimensions, we find in the $2 d$ case

$$
g^{2} \approx \frac{4 N_{c} \sigma_{\text {latt }}}{3 a^{2}}
$$

For $N_{c}=2$ and using the continuum value $\sqrt{\sigma_{\text {latt }}} / a \approx 0.44 \mathrm{GeV}$ we obtain $g^{2} \approx 0.516 \mathrm{GeV}^{2}$. 
One can check that, in the $2 d$ case, the functions $F_{1}\left(p^{2}\right)$ in Eq. (49) and $F_{2}\left(p^{2}\right)$ in Eq. (50) are again numerically indistinguishable. Note that $\sigma_{2 d}\left(\bar{\mu}^{2}\right) \approx 0.00179$ and $\sigma_{2 d}\left(p^{2}\right)$ takes values in $[0.00173,0.0334]$ when $p \in[0.018,2.553] \mathrm{GeV}$, which is the momentum interval for which we have numerically evaluated the ghost propagator $G\left(p^{2}\right)$. Thus, also in this case, the one-loop expression $p^{2} F_{1}\left(p^{2}\right)$ does not change appreciably in the considered momentum range. (It goes from 1.00 in the UV to 1.03 in the IR regimes.) As a consequence, the momentum dependence of the analytic prediction is entirely due to the factor $1 / p^{2}$ and in this case we should not expect a good description of the data in the IR region.

As in the $3 d$ and $4 d$ cases, the analytic prediction gives a good description of the data in the UV limit (see left plot in Fig. 14). However, since the value obtained for $g^{2}$ is smaller than the critical value $g_{c}^{2}$-i.e. the one-loop result is free-like at small momenta, while the numerical data are IR-enhanced - in the $2 d$ case one cannot indeed describe well the IR data by a simple global rescaling of the function $F_{1}\left(p^{2}\right)$ (see right plot in Fig. 14). On the other hand, by fitting $g^{2}$-i.e. considering the function $\widetilde{F}_{1}\left(p^{2}\right)$ - one finds that the value $g^{2}=13.46(2) \mathrm{GeV}^{2}$ allows a good description of the lattice data (see left plot in Fig. 15) with $\chi^{2} /$ dof $\approx 1.6$ and 320 data points. Let us stress that for $d=2$ choosing the fitted value for $g^{2}$ over the fixed theoretical one has a dramatic effect on the behavior of $p^{2} \widetilde{F}_{1}\left(p^{2}\right)$. Indeed, this quantity goes from about 1.0 at the largest momenta to about 9.0 in the IR limit.

Also, a slightly better fit can be obtained with the function ${ }^{11}$

$$
F_{2 d}\left(p^{2}\right)=\frac{z}{p^{2}}\left(\frac{1+p^{2} / s^{2}}{p^{2} / s^{2}}\right)^{t}
$$

Indeed, with the fitting parameters set to

$$
\begin{aligned}
& z=0.963 \pm 0.002 \\
& t=0.188 \pm 0.002 \\
& s=1.08 \pm 0.04 \mathrm{GeV}
\end{aligned}
$$

we find $\chi^{2} /$ dof $\approx 1.2$ (again with 320 data points). The corresponding plot is shown in Fig. 15 (right plot). Note that the factor $\left(s^{2} / p_{\text {min }}^{2}\right)^{t} \approx 4.7$ is compatible with the multiplicative constant obtained above when comparing the IR and UV behaviors of $F_{1}\left(p^{2}\right)$ (see right plot in Fig. 14).

As in the $3 d$ and $4 d$ cases, one can also estimate what is missing in the one-loop analysis, i.e. we can evaluate $W\left(p^{2}\right)$ [see Eqs. (58) and (59), using a Monte Carlo analysis with 10000 samples] as a function of the (unimproved) momenta $p$. The corresponding data (see Fig. 16) can be reasonably described by the fitting function $\widetilde{W}\left(p^{2}\right)$ [see Eq. (60)] with the fitting parameters

$$
A \approx 0.68, \quad B \approx 12.0 \mathrm{GeV}^{-2},
$$

which yields a $\chi^{2} / d o f$ of 2.5 (with 320 data points). It is also interesting to note that, in this case, as for $d=3$, the magnitude of what is missing in the one-loop calculation of $\sigma\left(p^{2}\right)$ is quite large, since $p^{2} F_{1}\left(p^{2}\right)$ is essentially constant.

\section{CONCLUSIONS}

We have presented the final step of our analysis of large-lattice Landau-gauge propagators as compared to predictions of the RGZ approach. Our data for the $\mathrm{SU}(2)$ ghost propagator $G\left(p^{2}\right)$ in $d=3,4$ and 2 have been compared first to the "direct" one-loop formulae, using the parameters from the gluon-propagator fits reported in [1] and a fixed (theoretical) value for the bare coupling $g^{2}$. This comparison is shown in Figs. 6, 10 and 14 respectively for $d=3,4$ and 2. In all cases we show the data (multiplicatively) normalized to $1 / \bar{\mu}^{2}$ for $p=\bar{\mu}=2.5 \mathrm{GeV}$. The proposed (one-loop) behavior is shown with the same normalization on the left side of the figures and, in all cases, there is a good description in the UV limit. On the right side of these figures, we have fixed the analytic form to match the numerical result at the smallest nonzero (lattice) momentum for the

\footnotetext{
${ }^{11}$ This fitting function is inspired by the one considered in Ref. [15] for the $2 d$ case, but with one less parameter. We have checked that the function employed in Ref. [15] allows only a modest improvement in the description of the data when compared to the simpler fitting function $F_{2 d}\left(p^{2}\right)$ considered here.
} 

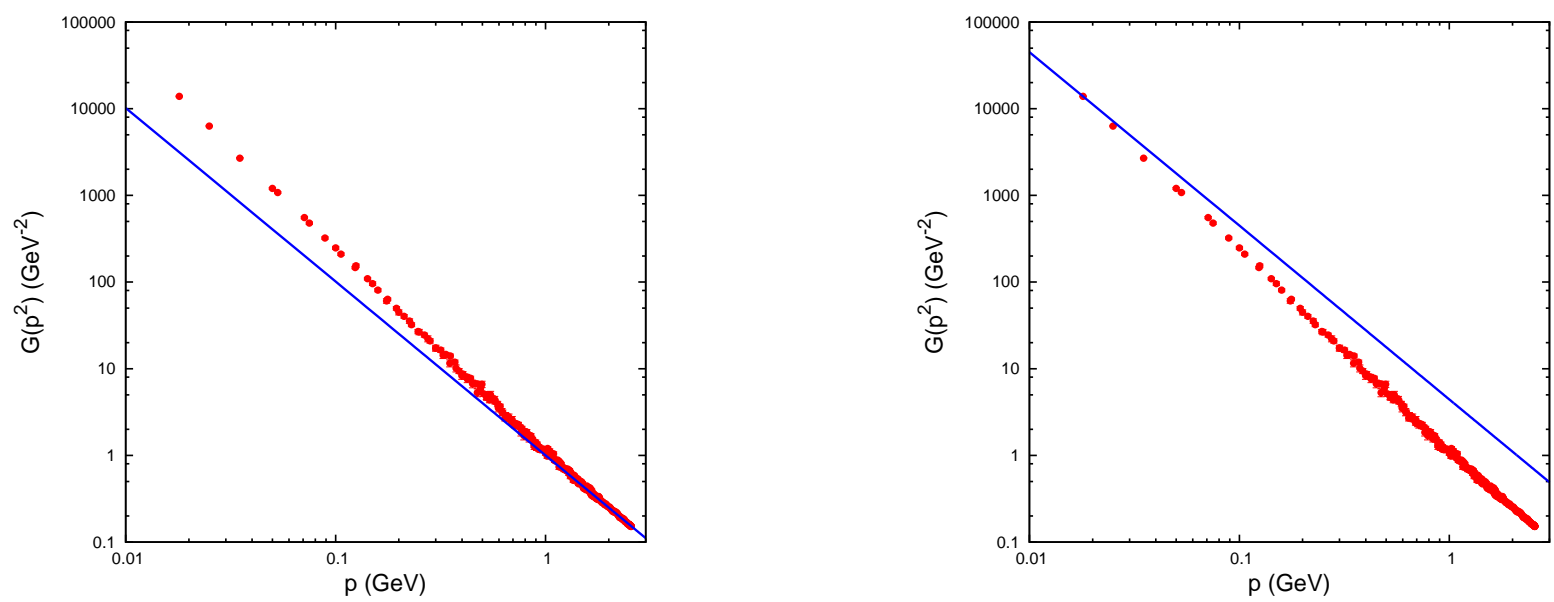

FIG. 14. Plot of the ghost propagator $G\left(p^{2}\right)$ as a function of the magnitude of the (unimproved) momenta $p$ (both in physical units) for the lattice volume $V=320^{2}$ at $\beta=10.0$. The data are (multiplicatively) normalized to $1 / \bar{\mu}^{2}$ for $p=\bar{\mu}=2.5 \mathrm{GeV}$. We also show the function $F_{1}\left(p^{2}\right)$ [see Eq. (49)] (normalized in the same way) with the Gribov ghost form factor $\sigma\left(p^{2}\right)$ given by the one-loop results (38)-(44); the corresponding parameters are reported in Eq. (70) and we set $g^{2}=0.516 \mathrm{GeV}^{2}$. On the other hand, in the right plot, we fix the analytic form to match the numerical result at $p=p_{\min }=18 \mathrm{MeV}$, the smallest nonzero (lattice) momentum for the pair $(V, \beta)$ considered, yielding $4.41862 F_{1}\left(p^{2}\right)$. Notice the logarithmic scale on both axes.
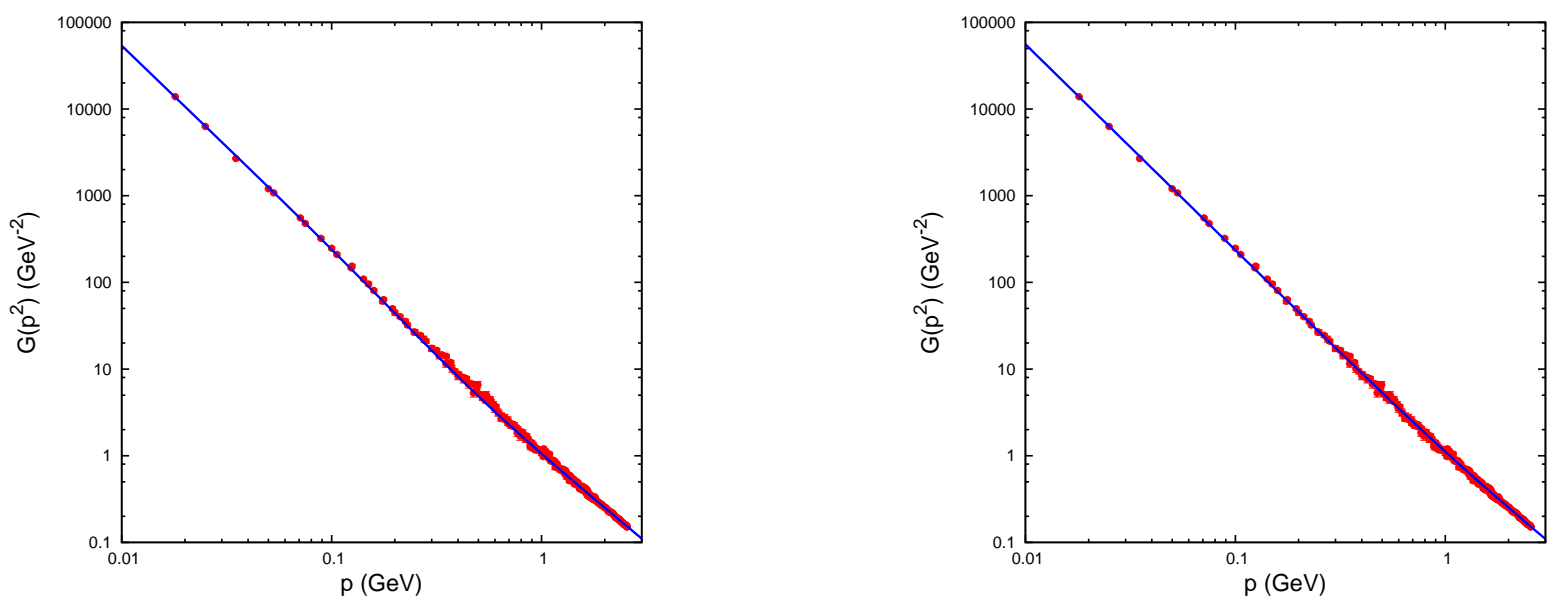

FIG. 15. Plot of the ghost propagator $G\left(p^{2}\right)$ as a function of the magnitude of the (unimproved) momenta $p$ (both in physical units) for the lattice volume $V=320^{2}$ at $\beta=10.0$ together with the fitting forms discussed in the text. The data are (multiplicatively) normalized to $1 / \bar{\mu}^{2}$ for $p=\bar{\mu}=2.5 \mathrm{GeV}$. In the left plot we show the function $\widetilde{F}_{1}\left(p^{2}\right)$ (normalized in the same way) with the Gribov ghost form factor $\sigma\left(p^{2}\right)$ given by the one-loop results (38)-(44); the corresponding parameters are reported in Eq. (70) and we use the fitted value $13.4556 \mathrm{GeV}^{2}$ for $g^{2}$. On the other hand, in the right plot, we show the fitting function $F_{2 d}\left(p^{2}\right)$ [see Eq. (73)] with the parameters given in Eqs. (74)-(76). Notice the logarithmic scale on both axes.

considered lattice volume and $\beta$ value, i.e. we plot a global rescaling of the one-loop prediction. We find that a good description of the IR region is obtained in $3 d$ and $4 d$, confirming that the IR behavior of $G\left(p^{2}\right)$ in these cases is simply enhanced by a factor with respect to the UV one. On the contrary, such a rescaling does not hold in $d=2$, since $G\left(p^{2}\right)$ is IR-enhanced in this case. This difference in IR behavior is clearly seen in Fig. 13, where we show the Gribov ghost form factor $\sigma\left(p^{2}\right)$ [see Eq. (2)] for the lattice volumes $V=320^{2}, 320^{3}$ and $128^{4}$ (respectively the largest volumes for each dimension $d$ ) as a function of the unimproved momenta [see Eq. (48)]. In particular, one clearly sees that in the $3 d$ and $4 d$ cases the Gribov ghost form factor becomes almost constant at small momenta. 


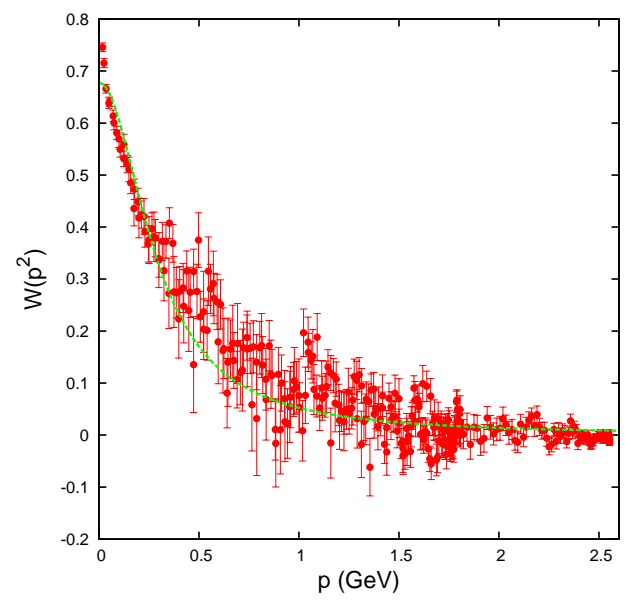

FIG. 16. Plot of the term $W\left(p^{2}\right)$ [see Eqs. (58) and (59)] as a function of the (unimproved) momenta $p$ (in physical units) for the lattice volume $V=320^{2}$ at $\beta=10.0$ (data points). We also show (in red) the fitting function $\widetilde{W}\left(p^{2}\right)$ [see Eq. (60)] with the fitting parameters reported in Eq. (77).

Next, we have shown the data as compared to the fitted one-loop prediction, i.e. we have used the same parameters as above, but fitting the value of the bare coupling $g^{2}$ to the data. A good description is obtained, with reasonable values of $\chi^{2} / d o f$ (respectively 4.5, 1.7 and 1.6 for $d=3,4$ and 2), as seen in the left-hand side of Figs. 8, 11 and 15 respectively for $d=3,4$ and 2. We note that an even better description (respectively with $\chi^{2} / d o f$ of $2.9,0.75$ and 1.2 for $d=3,4$ and 2 ) is obtained by fitting the function in Eq. (54) for $d=3,4$ and in Eq. (73) for $d=2$, as can be seen in the plots on the right in the same figures. The fact that one can describe well the whole range of data by using the analytic prediction for $G\left(p^{2}\right)$ with a fitted value for $g^{2}$ is an indication of the importance of having a one-parameter family of solutions for the propagators in $\mathrm{SU}\left(N_{c}\right)$ Yang-Mills theories (see Section I). We remark that the ratio between the fitted value of $g^{2}$ and the fixed theoretical value is found to be quite large ${ }^{12}$ in all three cases considered, namely it is about 7.7, 1.9 and 26 respectively for $d=3,4$ and 2 .

Finally, we have isolated the difference between the nonperturbative data and the one-loop results, by defining the function $W\left(p^{2}\right)$ in Eq. (58). As seen in Figs. 9, 12 and 16 respectively for $d=3,4$ and 2, this difference is small in the UV region and grows in the IR region. Moreover, the behavior of $W\left(p^{2}\right)$ is very similar in the three cases and, indeed, it may be reasonably well parametrized by a simple function of the momentum [see Eq. (60)]. This supports a unified explanation for the inaccuracy of the one-loop predictions in the IR region for the three cases. By considering the similar studies carried out in Refs. [69-71] in $d=4$, it is reasonable to assume that the use of a fully nonperturbative gluon propagator $D\left(p^{2}\right)$ in the one-loop analysis for $G\left(p^{2}\right)$ is not sufficient if one does not also use an improved ghost-gluon vertex. A detailed study of this vertex will be presented elsewhere [100].

\section{ACKNOWLEDGMENTS}

A.C. and T.M. acknowledge partial support from CNPq, while N.V. acknowledges the financial support from the Research Foundation-Flanders (FWO). Fits have been done using gnuplot 4.6 and python 2.7.6. In particular, in the $2 d$ case, when we need to consider the incomplete Beta function with complex argument we used a python code employing optimize.leastsq and mpmath. betainc. We have checked that, when the fits could be done both with gnuplot and our python code, we obtained

\footnotetext{
${ }^{12}$ A large value of $g^{2}$ has also been obtained in Refs. [55, 56] by fitting $4 d$ and $3 d$ lattice data for the gluon and the ghost propagators to analytic predictions obtained using a particular case of the Curci-Ferrari model [99].
} 
the same results.

[1] A. Cucchieri, D. Dudal, T. Mendes and N. Vandersickel, Phys. Rev. D85, 094513 (2012).

[2] R. Alkofer and L. von Smekal, Phys. Rept. 353, 281 (2001).

[3] J. Greensite, Lect. Notes Phys. 821, 1 (2011).

[4] I. C. Cloet and C. D. Roberts, Prog. Part. Nucl. Phys. 77, 1 (2014).

[5] N. Brambilla et al., Eur. Phys. J. C74, no. 10, 2981 (2014).

[6] D. Binosi and J. Papavassiliou, Phys. Rept. 479, 1 (2009).

[7] A. Cucchieri and T. Mendes, PoS QCD-TNT09, 026 (2009).

[8] P. Boucaud, J. P. Leroy, A. L. Yaouanc, J. Micheli, O. Pene and J. Rodriguez-Quintero, Few Body Syst. 53, 387 (2012).

[9] N. Vandersickel and D. Zwanziger, Phys. Rept. 520, 175 (2012).

[10] A. Maas, Phys. Rept. 524, 203 (2013).

[11] I. L. Bogolubsky, E. M. Ilgenfritz, M. Muller-Preussker and A. Sternbeck, PoS LAT2007, 290 (2007).

[12] A. Cucchieri and T. Mendes, PoS LAT2007, 297 (2007).

[13] A. Sternbeck, L. von Smekal, D. B. Leinweber and A. G. Williams, PoS LAT2007, 340 (2007).

[14] A. Cucchieri and T. Mendes, Phys. Rev. Lett. 100, 241601 (2008).

[15] A. Cucchieri and T. Mendes, Phys. Rev. D78, 094503 (2008).

[16] I. L. Bogolubsky, E. M. Ilgenfritz, M. Muller-Preussker and A. Sternbeck, Phys. Lett. B676, 69 (2009).

[17] V. G. Bornyakov, V. K. Mitrjushkin and M. Muller-Preussker, Phys. Rev. D81, 054503 (2010).

[18] A. Cucchieri, D. Dudal, T. Mendes and N. Vandersickel, PoS QCD-TNT-II, 030 (2012).

[19] O. Oliveira and P. J. Silva, Phys. Rev. D86, 114513 (2012).

[20] V. G. Bornyakov, E. M. Ilgenfritz, C. Litwinski, V. K. Mitrjushkin and M. Muller-Preussker, Phys. Rev. D92, no. 7, 074505 (2015).

[21] V. G. Bornyakov, V. K. Mitrjushkin and R. N. Rogalyov, Phys. Rev. D89, 054504 (2014).

[22] A. Maas, Phys. Rev. D75, 116004 (2007).

[23] A. Cucchieri and T. Mendes, AIP Conf. Proc. 1343, 185 (2011).

[24] A. C. Aguilar and A. A. Natale, JHEP 0408, 057 (2004).

[25] A. C. Aguilar and J. Papavassiliou, JHEP 0612, 012 (2006).

[26] A. C. Aguilar and J. Papavassiliou, Eur. Phys. J. A31, 742 (2007).

[27] A. C. Aguilar, D. Binosi and J. Papavassiliou, Phys. Rev. D78, 025010 (2008).

[28] A. C. Aguilar and J. Papavassiliou, Phys. Rev. D81, 034003 (2010).

[29] A. C. Aguilar, D. Binosi and J. Papavassiliou, Phys. Rev. D84, 085026 (2011).

[30] A. C. Aguilar, D. Binosi and J. Papavassiliou, Phys. Rev. D86, 014032 (2012).

[31] D. Binosi, D. Ibanez and J. Papavassiliou, Phys. Rev. D86, 085033 (2012).

[32] L. von Smekal, A. Hauck and R. Alkofer, Annals Phys. 267, 1 (1998) [Erratum-ibid. 269, 182 (1998)].

[33] D. Zwanziger, Phys. Rev. D65, 094039 (2002).

[34] C. Lerche and L. von Smekal, Phys. Rev. D65, 125006 (2002).

[35] C. S. Fischer, J. Phys. G32, R253 (2006).

[36] M. Q. Huber, R. Alkofer, C. S. Fischer and K. Schwenzer, Phys. Lett. B659, 434 (2008).

[37] R. Alkofer, M. Q. Huber and K. Schwenzer, Phys. Rev. D81, 105010 (2010).

[38] M. Q. Huber, A. Maas and L. von Smekal, JHEP 1211, 035 (2012).

[39] V. N. Gribov, Nucl. Phys. B139, 1 (1978).

[40] D. Zwanziger, Nucl. Phys. B323, 513 (1989).

[41] D. Zwanziger, Phys. Lett. B257, 168 (1991).

[42] D. Zwanziger, Nucl. Phys. B364, 127 (1991).

[43] D. Zwanziger, Nucl. Phys. B399, 477 (1993).

[44] D. Zwanziger, Nucl. Phys. B412, 657 (1994).

[45] M. A. L. Capri, D. Dudal, M. S. Guimaraes, L. F. Palhares and S. P. Sorella, Phys. Lett. B719, 448 (2013).

[46] D. Dudal, J. A. Gracey, S. P. Sorella, N. Vandersickel and H. Verschelde, Phys. Rev. D78, 065047 (2008).

[47] D. Dudal, J. A. Gracey, S. P. Sorella, N. Vandersickel and H. Verschelde, Phys. Rev. D78, 125012 (2008).

[48] D. Dudal, S. P. Sorella, N. Vandersickel and H. Verschelde, Phys. Lett. B680, 377 (2009). 
[49] S. P. Sorella, D. Dudal, M. S. Guimaraes and N. Vandersickel, PoS FACESQCD, 022 (2010).

[50] N. Vandersickel, D. Dudal and S. P. Sorella, PoS FACESQCD, 044 (2010).

[51] N. Vandersickel, [arXiv:1104.1315 [hep-th]].

[52] D. Dudal, S. P. Sorella and N. Vandersickel, Phys. Rev. D84, 065039 (2011).

[53] M. Frasca, Phys. Lett. B670, 73 (2008).

[54] K. -I. Kondo, Phys. Lett. B678, 322 (2009).

[55] M. Tissier and N. Wschebor, Phys. Rev. D82, 101701 (2010).

[56] M. Tissier and N. Wschebor, Phys. Rev. D84, 045018 (2011).

[57] A. Weber, Phys. Rev. D85, 125005 (2012).

[58] M. R. Pennington and D. J. Wilson, Phys. Rev. D84, 119901 (2011).

[59] F. J. Llanes-Estrada and R. Williams, Phys. Rev. D86, 065034 (2012).

[60] A. Weber and P. Dall'Olio, EPJ Web Conf. 80, 00016 (2014).

[61] M. Pelez, M. Tissier and N. Wschebor, Phys. Rev. D90, 065031 (2014).

[62] F. Siringo, arXiv:1509.05891 [hep-ph].

[63] F. A. Machado, arXiv:1601.02067 [hep-ph].

[64] A. Cucchieri, D. Dudal and N. Vandersickel, Phys. Rev. D85, 085025 (2012).

[65] P. Boucaud, J-P. Leroy, A. Le Yaouanc, J. Micheli, O. Pene and J. Rodriguez-Quintero, JHEP 0806, 012 (2008).

[66] P. Boucaud, J. P. Leroy, A. Le Yaouanc, J. Micheli, O. Pene and J. Rodriguez-Quintero, JHEP 0806, 099 (2008).

[67] C. S. Fischer, A. Maas and J. M. Pawlowski, Annals Phys. 324, 2408 (2009).

[68] J. Rodriguez-Quintero, JHEP 1101, 105 (2011).

[69] D. Dudal, O. Oliveira and J. Rodriguez-Quintero, Phys. Rev. D86, 105005 (2012).

[70] A. C. Aguilar, D. Ibañez and J. Papavassiliou, Phys. Rev. D87, 114020 (2013).

[71] A. C. Aguilar, PoS QCD-TNT-III, 001 (2013).

[72] M. Q. Huber and L. von Smekal, JHEP 1304, 149 (2013).

[73] “Table of Integrals, Series, and Products", I. S. Gradshteyn and I. M. Ryzhik, Edited by Alan Jeffrey and Daniel Zwillinger (Academic Press, 2007), 7th ed.

[74] A. Cucchieri, T. Mendes and A. R. Taurines, Phys. Rev. D67, 091502 (2003).

[75] J. C. R. Bloch, A. Cucchieri, K. Langfeld and T. Mendes, Nucl. Phys. B687, 76-100 (2004).

[76] A. Cucchieri and T. Mendes, Nucl. Phys. B471, 263-292 (1996).

[77] A. Cucchieri, Nucl. Phys. B508, 353 (1997).

[78] P. J. Silva and O. Oliveira, Nucl. Phys. B690, 177 (2004).

[79] I. L. Bogolubsky, G. Burgio, M. Muller-Preussker and V. K. Mitrjushkin, Phys. Rev. D74, 034503 (2006).

[80] I. L. Bogolubsky, E. M. Ilgenfritz, M. Muller-Preussker and A. Sternbeck, PoS LATTICE2009, 237 (2009).

[81] A. Maas, J. M. Pawlowski, D. Spielmann, A. Sternbeck, L. von Smekal, Eur. Phys. J. C68, 183-195 (2010).

[82] A. Sternbeck and M. Muller-Preussker, Phys. Lett. B726, 396 (2013).

[83] P. Boucaud et al., Nucl. Phys. Proc. Suppl. 106, 266 (2002).

[84] S. Furui and H. Nakajima, Few Body Syst. 40, 101 (2006).

[85] E. M. Ilgenfritz, M. Muller-Preussker, A. Sternbeck, A. Schiller and I. L. Bogolubsky, Braz. J. Phys. 37, 193 (2007).

[86] P. O. Bowman et al., Phys. Rev. D76, 094505 (2007).

[87] P. J. Silva and O. Oliveira, PoS LATTICE2010, 287 (2010).

[88] P. Boucaud et al., Phys. Rev. D72, 114503 (2005).

[89] A. Cucchieri, A. Maas and T. Mendes, Phys. Rev. D74, 014503 (2006).

[90] A. Cucchieri, T. Mendes and A. Mihara, Phys. Rev. D72, 094505 (2005).

[91] J. P. Ma, Mod. Phys. Lett. A15, 229 (2000).

[92] D. B. Leinweber, J. I. Skullerud, A. G. Williams and C. Parrinello [UKQCD Collaboration], Phys. Rev. D60, 094507 (1999) [Erratumibid. D61, 079901 (2000)].

[93] F. de Soto and C. Roiesnel, JHEP 0709, 007 (2007).

[94] B. Lucini, M. Teper, Phys. Rev. D66, 097502 (2002).

[95] W. Celmaster and R. J. Gonsalves, Phys. Rev. D20, 1420 (1979).

[96] P. Boucaud et al., Phys. Rev. D79, 014508 (2009).

[97] B. Lucini and G. Moraitis, Phys. Lett. B668, 226 (2008).

[98] H. G. Dosch, V. F. Muller, Fortsch. Phys. 27, 547 (1979).

[99] G. Curci and R. Ferrari, Nuovo Cim. A32, 151 (1976). 
[100] A. Cucchieri and T. Mendes, in preparation. 